\title{
Article
}

\section{Hazelnut Kernel Size and Industrial Aptitude}

\author{
Agusti Romero-Aroca $\left.{ }^{1, *} \mathbb{(}\right)$, Mercè Rovira ${ }^{1}$, Valerio Cristofori ${ }^{2} \mathbb{B}$ and Cristian Silvestri ${ }^{2} \mathbb{C}$ \\ 1 Institut de Recerca i Tecnologia Agroalimentàries IRTA-Mas Bové, Ctra. Reus-El Morell km 3.8, \\ 43120 Constantí, Spain; merce.rovira@irta.cat \\ 2 Dipartimento di Scienze Agrarie e Forestali (DAFNE), Università degli Studi della Tuscia, \\ Via S. Camillo de Lellis snc, 01100 Viterbo, Italy; valerio75@unitus.it (V.C.); silvestri.c@unitus.it (C.S.) \\ * Correspondence: agusti.romero@irta.cat
}

Citation: Romero-Aroca, A.; Rovira, M.; Cristofori, V.; Silvestri, C. Hazelnut Kernel Size and Industrial Aptitude. Agriculture 2021, 11, 1115. https://doi.org/10.3390/ agriculture11111115

Academic Editor: Fabio Sciubba

Received: 20 September 2021

Accepted: 5 November 2021

Published: 9 November 2021

Publisher's Note: MDPI stays neutral with regard to jurisdictional claims in published maps and institutional affiliations.

Copyright: (c) 2021 by the authors. Licensee MDPI, Basel, Switzerland. This article is an open access article distributed under the terms and conditions of the Creative Commons Attribution (CC BY) license (https:// creativecommons.org/licenses/by/ $4.0 /)$.

\begin{abstract}
Kernel size is the main hazelnut quality parameter for the consumption market. However, industrial purposes are the main destination for the main hazelnut cultivars. This work aims to identify industrial aptitude relationships to kernel size, and qualitative nut and kernel traits eligible to enhance hazelnut's commercial value. The qualitative hazelnut traits of cv "Negret" and "Pauetet" were assessed via in-shell and shelled nut sizes for two years. In-shell hazelnuts were tested for weight, shape, percent kernel, yield and shell thickness. Kernels were measured for shape, weight, roasting aptitude, skin color, moisture content and water activity, free acidity, fat content, crude protein, total sugars, minerals, fatty acid composition, $\alpha$-tocopherol and oil stability. In-shell hazelnut traits significantly differed between cultivars, sizes and storage period. Shell thickness and nut roundness increased almost linearly with nut caliber, whereas kernel percentage decreased. Kernel roundness increased linearly with caliber. The blanching and roasting aptitude of "Negret" increased linearly with caliber, whereas no significant trend was observed for "Pauetet". Significant differences between cultivars were confirmed for water activity, oil acidity and skin color. Regarding chemical composition, fat content increased linearly with caliber in both cultivars, and $\alpha$-tocopherol followed the same trend in "Negret". The sugar content tended to decrease with caliber, whereas crude protein, fiber and minerals did not show any significant relationship with kernel size. Unsaturated and polyunsaturated fatty acids fitted to linear models related to caliber, showing differences between cultivars. Unsaturated fatty acids increased with caliber, whereas polyunsaturated fatty acids decreased, and the oil stability increased linearly with kernel size. These results show that some hazelnut key traits change significantly with kernel size. The linear models presented could be a powerful tool for the confectionery industry to modulate the industrial value of given hazelnut batches.
\end{abstract}

Keywords: fruit caliber; fruit quality; industrial aptitude; fatty acids; fat content; nut shape

\section{Introduction}

Hazelnuts are considered a typical food of the Mediterranean diet, and their acceptance is growing in the international market [1]. Raw consumption is less than $10 \%$, whereas industrial uses account for $90 \%$. The aim is to provide the confectionery industries, cooperatives and first transformers with process kernels to produce slices, sticks, dices, flour, paste or whole kernels, among other specialties. The chocolate industry is the main hazelnut user $(60-70 \%)$, though other confectioneries are relevant as well, such as nougat, marzipan, ice cream, cakes, beverages, praline and spreadable creams [2,3].

The industrial requirements of the supplied batches of hazelnuts are becoming increasingly exigent. The kernel size and homogeneity as industrial traits have been very important during the last forty years $[4,5]$. Since many confectionery applications use roasted hazelnuts, this process is one of the most studied. Liepe [6] described the taste changes that happen in an energy bar filled with different kinds of nuts and cereals. Voight et al. [7] concluded that a group of globular proteins from both cocoa beans and roasted nuts defined the final aroma of the chocolate. Ebrahem et al. [8] reported that the 
hazelnut storage temperature and the health status of the nuts affect their roasting aptitude and the final shelf-life of the product, while the importance of the roasting temperature was studied by Perren and Escher [9] and Richardson and Ebrahem [10], among others. The relationship among hazelnut cultivar, roasting intensity and consumer acceptability was analyzed by Romero et al. [11], while the kinetics of color change during the roasting process was described by Demir et al. [12]. Finally, the microstructural changes that happen during roasting were studied by Saklar et al. [13]. All these studies describe the interaction between processing conditions and some chemical compounds of hazelnuts. Thus, cultivar physical and chemical traits are a main factor to consider. There is available information about hazelnut characteristics and composition from many countries, such as Italy [14-16], Spain [2,17], USA [18], Turkey [19], Poland [20-23] and other European countries [24,25].

In Spain, the main hazelnut cultivars are "Negret" and "Pauetet" [26]. The selected clone "N-9" from cv "Negret" is the most planted in the Tarragona area, which is highly valued by the confectionery industry and has a very high roasting and blanching aptitude. "Pauetet" is important in the Tarragona area and in Southwest France [27], and is often mixed with "Negret", because both cultivars have a similar kernel shape [2], but "Pauetet" has a worse blanching aptitude [26]. However, both cultivars have a slightly different chemical composition [2,28,29].

Kernel size is a main hazelnut quality parameter for the consumption market. However, most hazelnut cultivars are used for industrial purposes. For industrial applications that use whole kernels, caliber has paramount relevance. Nevertheless, there is no information about the relationship between kernel size, within the same cultivar, and industrial aptitude when the kernel is sliced, diced, or grounded. Though inter-batch variations in the physical and chemical traits of a given cultivar have been explained as a function of geographical origin, irrigation conditions and environmental factors in a given year [30], little is known about intra-batch variations. In this regard, kernel size could be a source of variation that is assumed by the industry to a certain extent, but few studies have focused on this.

Xenia has been proposed as a mechanism affecting hazelnut kernel traits and nutritional composition [31], as it was observed in other nuts, such as almond [32-35], chestnut [36], pistachio [37] and macadamia [38]. All these studies suggest that xenia showed a qualitative effect, where cross-pollinated nuts grew and changed in some chemical components (i.e., oil content, fatty acids and tocopherol. Furthermore, these studies suggest that some cultivars affect kernel traits more than others. However, industrial experience suggest that more mechanisms could be involved, since it is suspected that differences are not exclusive to bigger calibers.

This work aimed to study if industrial aptitude is related to kernel size, which parameters are the most variable and how to improve grading strategies to enhance the commercial value of hazelnut.

\section{Materials and Methods}

\subsection{Plant Material}

Two hazelnut varieties were studied, "Negret" and "Pauetet", which are the most common in Spain. Samples (10 kg), both in-shell and shelled, were taken from COSELVA, a cooperative in La Selva del Camp (Spain), after calibration. The study was conducted in two consecutive years (2008-2009).

Six in-shell hazelnut sizes were considered: $12.5-14 \mathrm{~mm}, 14-14.5 \mathrm{~mm}, 14.5-15 \mathrm{~mm}$, 15.5-16 mm, 16-17 $\mathrm{mm}$ and over $17 \mathrm{~mm}$. Additionally, seven shelled hazelnut sizes were considered: below $9 \mathrm{~mm}, 9-10 \mathrm{~mm}, 10-11 \mathrm{~mm}, 11-12 \mathrm{~mm}, 12-13 \mathrm{~mm}$ and over $13 \mathrm{~mm}$. Finally, for both in-shell and shelled samples, the average width was measured for 50 nuts and considered as the mean size (caliber) for that sample.

\subsection{Physical Traits of In-Shell Nut}

From each sample, up to 50 nuts were randomly taken, and several measurements were performed. Fruit dimensions (length, width and thickness) were measured (Figure 1) with a 
caliper (Mitutoyo, Model Absolute AOS Digimatic, San Francisco Bay Area, CA, USA). Fruit roundness was computed from the following expression:

$$
\text { Roundness }=\frac{\text { width }+ \text { thickness }}{2 \times \text { length }}
$$

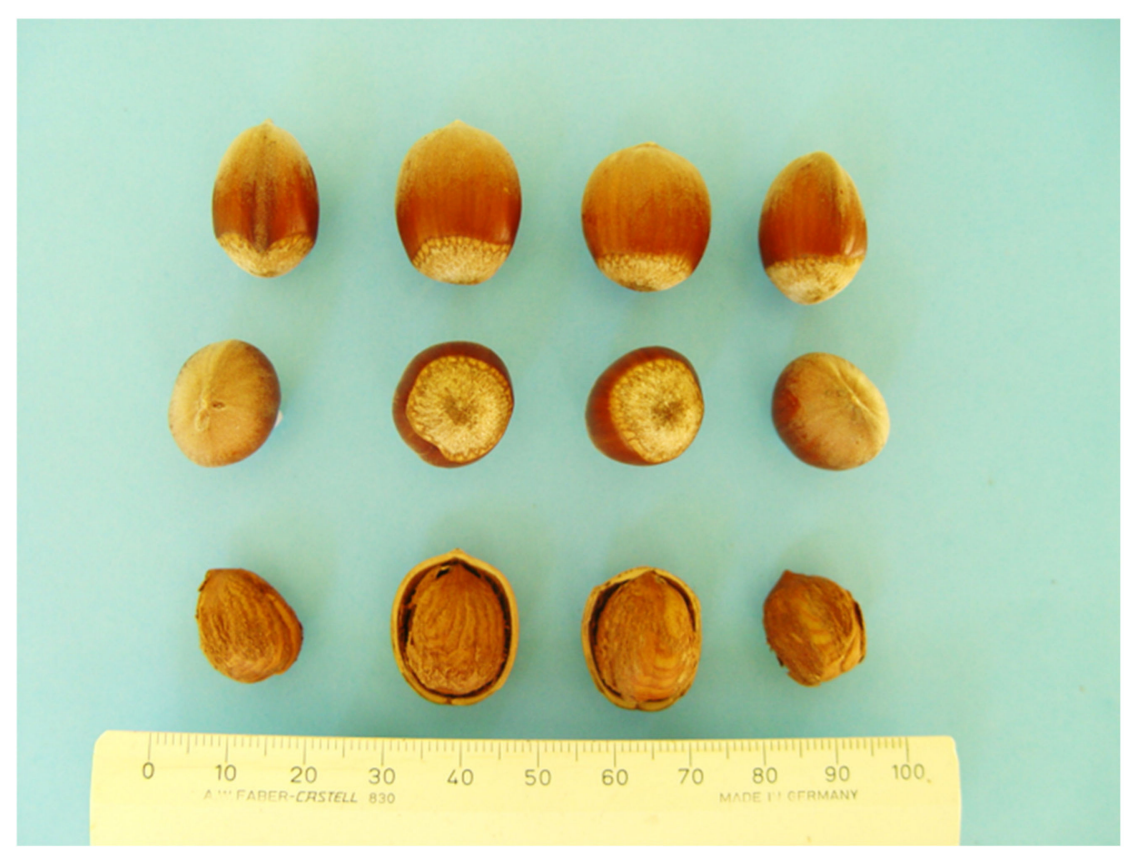

Figure 1. Hazelnut samples of $\mathrm{cv}$ "Negret" for its nut and kernel technological characterization.

Kernel yield (\%) was computed as follows:

$$
\text { Kernel yield }(\%)=\left(\frac{\text { weight of } 50 \text { shelled kernels }}{\text { weight of } 50 \text { nuts in shell }}\right) \times 100
$$

The thickness of the shell was measured on the convex side of each half using a digital caliper. Finally, the shell water content was expressed as the loss of weight (\%) in an oven at $105 \pm 1{ }^{\circ} \mathrm{C}$ to constant weight.

\subsection{Physical Traits of Shelled Nut}

Physical measurements were carried out on 50 kernels from each sample. Dimensions and roundness were measured as described above. Roasting indexes R1 (\% of nuts peeled in more than $95 \%$ ) and R2 (\% of nuts peeled in more than $50 \%$ ) were measured after keeping them in an oven (JP-SELECTA "dry big" model, using forced air) at $175 \pm 1{ }^{\circ} \mathrm{C}$ for $30 \mathrm{~min}$. Blanching indexes B1 (\% of nuts peeled in more than 95\%) and B2 (\% of nuts peeled in more than $50 \%$ ) were measured after keeping them in an oven at $115 \pm 1{ }^{\circ} \mathrm{C}$ for $20 \mathrm{~min}$.

Skin color components, lightness $\left(\mathrm{L}^{*}\right)$, reddish $\left(a^{*}\right)$ and yellowish $\left(b^{*}\right)$, were measured using a spectrophotometer (MINOLTA Model M3500) on fifty nuts for each variety and size. From these data, Chroma and Hue angles were determined using the following expressions:

$$
\text { Chroma }=\sqrt{\boldsymbol{a}^{* 2}+\boldsymbol{b}^{* 2}} \text { Hue angle }\left(^{\circ}\right)=\tan ^{-1}\left(\frac{\boldsymbol{b} *}{\boldsymbol{a} *}\right)
$$

\subsection{Chemical Composition}

Fat content was analyzed by the Soxhlet method, using 5-6 g of crushed kernels (with skin) and petroleum ether (boiling point 40 to $60^{\circ} \mathrm{C}$ ) for $7 \mathrm{~h}$ in the Soxhlet apparatus. Crude protein was analyzed by Dumas' combustion procedure using a Leco FP-528 analyzer [39]. Briefly, $0.2 \mathrm{~g}$ of grounded sample was weighed in a porcelain sample holder (boat) for introduction into the combustion chamber $\left(850 \pm 1{ }^{\circ} \mathrm{C}\right)$ utilizing an automated sample 
loader. The combustion process converts covalently bound nitrogen into nitrogen gas $\left(\mathrm{N}_{2}\right)$, which is quantified by passing the gas through a conductivity cell. The protein content was computed using a 6.25 factor. Crude fiber was measured using $1 \mathrm{~g}$ of ground sample by adding boiling $0.26 \mathrm{~N}$ sulfuric acid $(30 \mathrm{~min}$ ) followed by boiling $0.23 \mathrm{~N}$ potassium hydroxide $(30 \mathrm{~min})$. The extracted residue was dried at $103 \pm 1{ }^{\circ} \mathrm{C}(3 \mathrm{~h})$ and the dried sample weighed and placed into a furnace $\left(550 \pm 1{ }^{\circ} \mathrm{C}\right.$ for $3 \mathrm{~h}$ ); finally, the ashes were weighed. The mineral fraction was determined by burning $4 \mathrm{~g}$ of sample in a furnace at $550 \pm 1{ }^{\circ} \mathrm{C}$ for $4 \mathrm{~h} \mathrm{[40]}$.

The total sugar content was analyzed using the Luff-Schoorl method. For this, $2.5 \mathrm{~g}$ of crushed sample was extracted with ethanol $(1 \mathrm{~h})$, and then $5 \mathrm{~mL}$ of Carrez I and $5 \mathrm{~mL}$ of Carrez II solutions were added (1 min each). After ethanol evaporation, the extract was diluted in $200 \mathrm{~mL}$ of warm water to obtain a solution in which the total sugars after inversion were analyzed by the Luff-Schoorl method. Briefly, this consists of boiling $25 \mathrm{~mL}$ of solution for $10 \mathrm{~min}$, adding $10 \mathrm{~mL}$ of potassium iodide and $25 \mathrm{~mL}$ of $6 \mathrm{~N}$ sulfuric acid, and then titrating with $0.1 \mathrm{~N}$ sodium thiosulfate solution for neutralization. This value was compared with an equivalent solution, but not boiled.

Fatty acids were analyzed by gas-chromatography with a flame ionization detector (GC-FID) using a capillary column. The fatty acid methyl esters (FAMEs) were prepared by trans-esterification with $0.5 \mathrm{M}$ potassium hydroxide, following the official method UNE-EN ISO 5509:2000. FAMEs (1 mL) were separated using a gas-chromatograph (HP 6890; Agilent Technologies, Barcelona, Spain) equipped with an FID detector and a capillary column (30 $\mathrm{m} \times 0.25 \mathrm{~mm}$ i.d. (HP-Innowax, Agilent Technologies, Santa Clara, CA, USA)). The carrier gas was helium, and the flow rate was $1 \mathrm{~mL} \cdot \mathrm{min}^{-1}$. The injector and detector temperatures were 220 and $275^{\circ} \mathrm{C}$, respectively. The FAME identification was based on the retention time relative to that of a standard FAME mixture (Sigma-Aldrich, Madrid, Spain).

$\alpha$-tocopherol was measured by HPLC using a fluorescence detector (FLD), following the method Ce8-89 from AOCS. We used a PerkinElmer Series 200 HPLC-FLD (Shelton, CT, USA). Oil-free acidity was measured according to the AOAC method 16,239 [41]. Finally, oil stability was measured by the Rancimat method, using a Rancimat 617 series 09 Methrom, with a working temperature of $120^{\circ} \mathrm{C}$ and $20 \mathrm{~L} / \mathrm{h}$ of air flow. Oil was previously extracted from raw kernels using a manual press, filtered through a paper filter and stored in the freezer $\left(-20^{\circ} \mathrm{C}\right)$ until the analysis. All analyses were repeated twice.

\subsection{Statistical Analysis}

In the first approach, an analysis of covariance (ANCOVA) was performed considering both cultivar and time as the main factors, whereas nut size was used as a covariate. A linear model analysis was performed since, in all the cases, the covariate was highly significant (data not shown).

General Linear Model analysis was carried out to study if there was a significant linear relationship between nut size and each studied parameter and to determine if such linear model was equivalent for both cultivars. The GLM procedure from SAS software was used (version 9.4; SAS Institute, Cary, NC, USA).

\section{Results}

\subsection{In-Shell Hazelnuts}

The in-shell nut roundness index decreased with fruit caliber (Table S1), ranging from 1.29 to 1.19 for "Negret" and from 1.32 to 1.16 for "Pauetet" (Figure 2). Thus, it can be stated that bigger fruits have a more spherical shape for the same cultivar, which is a desirable characteristic for industrial shell-removing operations. Both cultivars showed the same trend, which fits very well to a linear model $\left(R^{2}>0.87 ; p<0.002\right)$, without statistical differences between cultivars regarding the slope of the model (Table 1). As expected, the mean roundness indexes for both cultivars were not significantly different. 


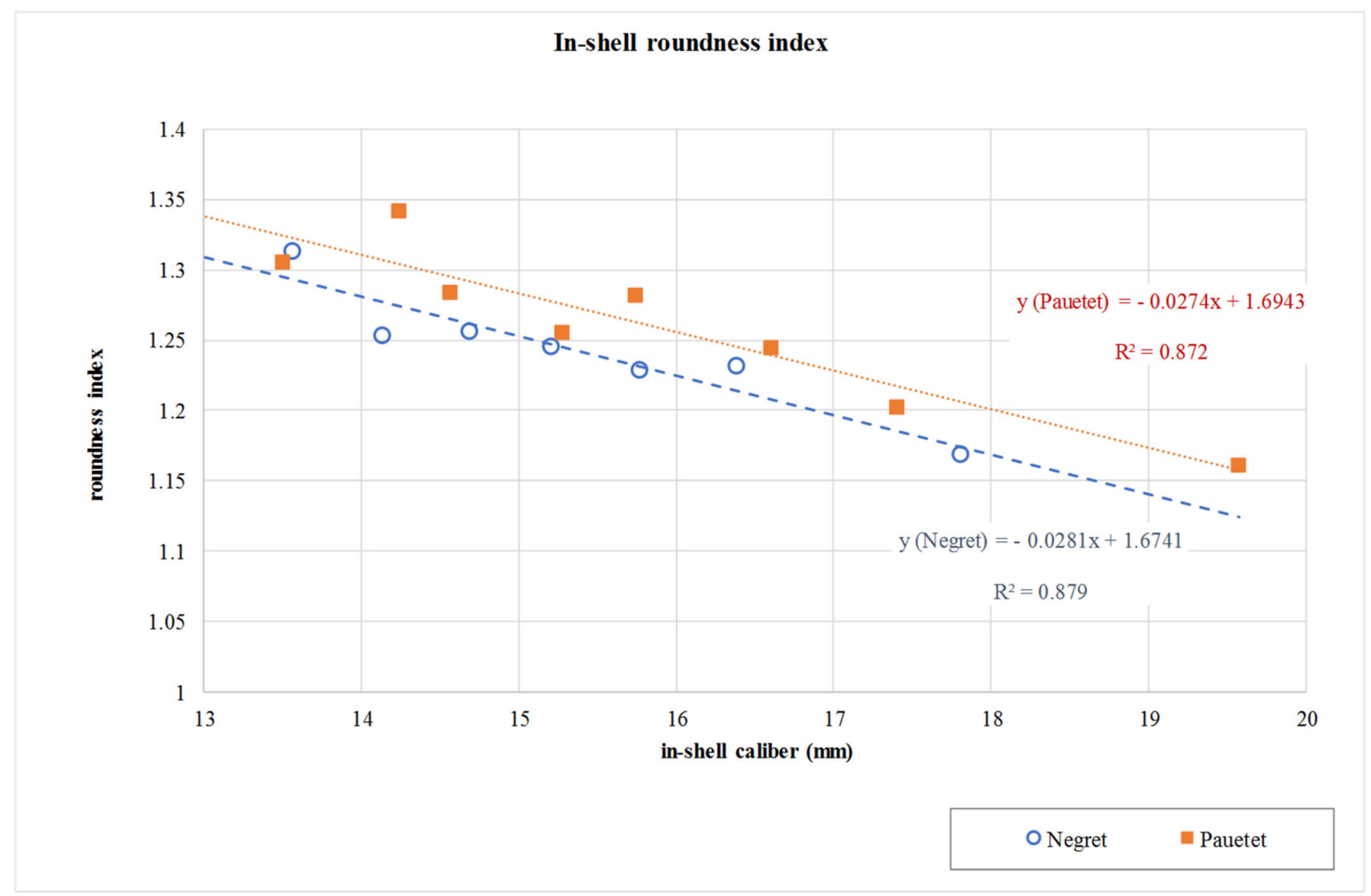

Figure 2. Roundness index linear models for in-shell hazelnuts of cv "Negret" and "Pauetet".

Table 1. Hazelnut in-shell nut traits of cv "Negret" and "Pauetet". Interpolated linear models and statistical tests for slope and cultivar homogeneity.

\begin{tabular}{|c|c|c|c|c|c|c|c|c|c|c|c|c|c|}
\hline \multirow{2}{*}{ Nut Trait } & \multirow[t]{2}{*}{ Cultivar } & \multicolumn{8}{|c|}{ Linear Models } & \multicolumn{2}{|c|}{ Test for Slope } & \multicolumn{2}{|c|}{ Test for Cultivar } \\
\hline & & \multicolumn{3}{|c|}{ Intercept } & \multicolumn{2}{|c|}{ Slope } & \multirow{2}{*}{$\begin{array}{l}\mathbf{R}^{2} \\
0.895\end{array}$} & \multirow{2}{*}{$\begin{array}{c}\text { F Model } \\
42.44\end{array}$} & \multirow{2}{*}{$\begin{array}{c}\text { P Model } \\
0.0013 \\
\end{array}$} & \multirow{3}{*}{$\begin{array}{c}\text { F Slope } \\
2.70\end{array}$} & \multirow{3}{*}{$\begin{array}{c}\text { P Slope } \\
0.1283\end{array}$} & \multirow{3}{*}{$\begin{array}{c}\text { F Cultivar } \\
41.07\end{array}$} & \multirow{3}{*}{$\begin{array}{l}\text { P Cultivar } \\
<0.0001\end{array}$} \\
\hline \multirow{2}{*}{ Kernel yield (\%) } & Negret & 57.17 & \pm & 1.53 & $-0.6466 \pm$ & 0.0993 & & & & & & & \\
\hline & Pauetet & 54.85 & \pm & 1.69 & $-0.3815 \pm$ & 0.1062 & 0.683 & 12.92 & 0.0114 & & & & \\
\hline \multirow{2}{*}{$\begin{array}{l}\text { Thickness of the } \\
\text { shell }(\mathrm{mm})\end{array}$} & Negret & -0.043 & \pm & 0.118 & 0.0622 & 0.0077 & 0.929 & 65.08 & 0.0005 & \multirow{2}{*}{2.17} & \multirow{2}{*}{0.1684} & \multirow{2}{*}{0.23} & \multirow{2}{*}{0.6387} \\
\hline & Pauetet & 0.186 & \pm & 0.117 & 0.0452 & 0.0074 & 0.862 & 37.49 & 0.0009 & & & & \\
\hline \multirow{2}{*}{ Shell moisture (\%) } & Negret & 14.32 & \pm & 1.04 & $-0.0297 \pm$ & 0.0678 & 0.037 & 0.19 & 0.6799 & \multirow{2}{*}{3.69} & \multirow{2}{*}{0.0808} & \multirow{2}{*}{16.91} & \multirow{2}{*}{0.0017} \\
\hline & Pauetet & 11.04 & \pm & 1.50 & 0.2310 & 0.0942 & 0.500 & 6.01 & 0.0497 & & & & \\
\hline \multirow{2}{*}{$\begin{array}{l}\text { In-shell length } \\
\quad(\mathrm{mm})\end{array}$} & Negret & 6.899 & \pm & 0.820 & 0.6984 & 0.0533 & 0.972 & 171 & $<0.0001$ & \multirow{2}{*}{0.49} & \multirow{2}{*}{0.4977} & \multirow{2}{*}{30.58} & \multirow{2}{*}{0.0002} \\
\hline & Pauetet & 8.334 & \pm & 0.982 & 0.6340 & 0.0615 & 0.947 & 106 & $<0.0001$ & & & & \\
\hline \multirow{2}{*}{$\begin{array}{l}\text { In-shell thickness } \\
\qquad(\mathrm{mm})\end{array}$} & Negret & 0.910 & \pm & 0.514 & 0.7930 & 0.0334 & 0.991 & 565 & $<0.0001$ & \multirow{2}{*}{2.03} & \multirow{2}{*}{0.1821} & \multirow{2}{*}{44.51} & \multirow{2}{*}{$<0.0001$} \\
\hline & Pauetet & 1.191 & \pm & 0.403 & 0.7319 & 0.0253 & 0.993 & 840 & $<0.0001$ & & & & \\
\hline In-shell roundness & Negret & 1.674 & \pm & 0.071 & $-0.0281 \pm$ & 0.0047 & 0.879 & 36.36 & 0.0018 & & & & \\
\hline$(\mathrm{mm})$ & Pauetet & 1.694 & \pm & 0.068 & $-0.0274 \pm$ & 0.0043 & 0.873 & 41.19 & 0.0007 & 0.01 & 0.9204 & 0.04 & 0.8320 \\
\hline
\end{tabular}

Concerning in-shell nut dimensions, both length and thickness increased linearly with nut caliber (width). These results fit very well to a linear model (Table 1 ) for both dimension traits $\left(R^{2}>0.94\right.$ for length and $R^{2}>0.99$ for thickness). However, thickness increases faster than length. This can explain the trend of nuts being more spherical at a higher nut size. Even though nuts of cv "Pauetet" were significantly longer and thicker than those of the $\mathrm{cv}$ "Negret", no significant differences for linear model slopes were observed.

The thickness of the shell measured at the equatorial cross section was wider at higher calibers. A significant linear trend was observed between both traits $\left(R^{2}>0.86 ; p<0.0001\right)$, without any significant difference between cultivars either for slope or mean values (Table 1 and Figure 3). 


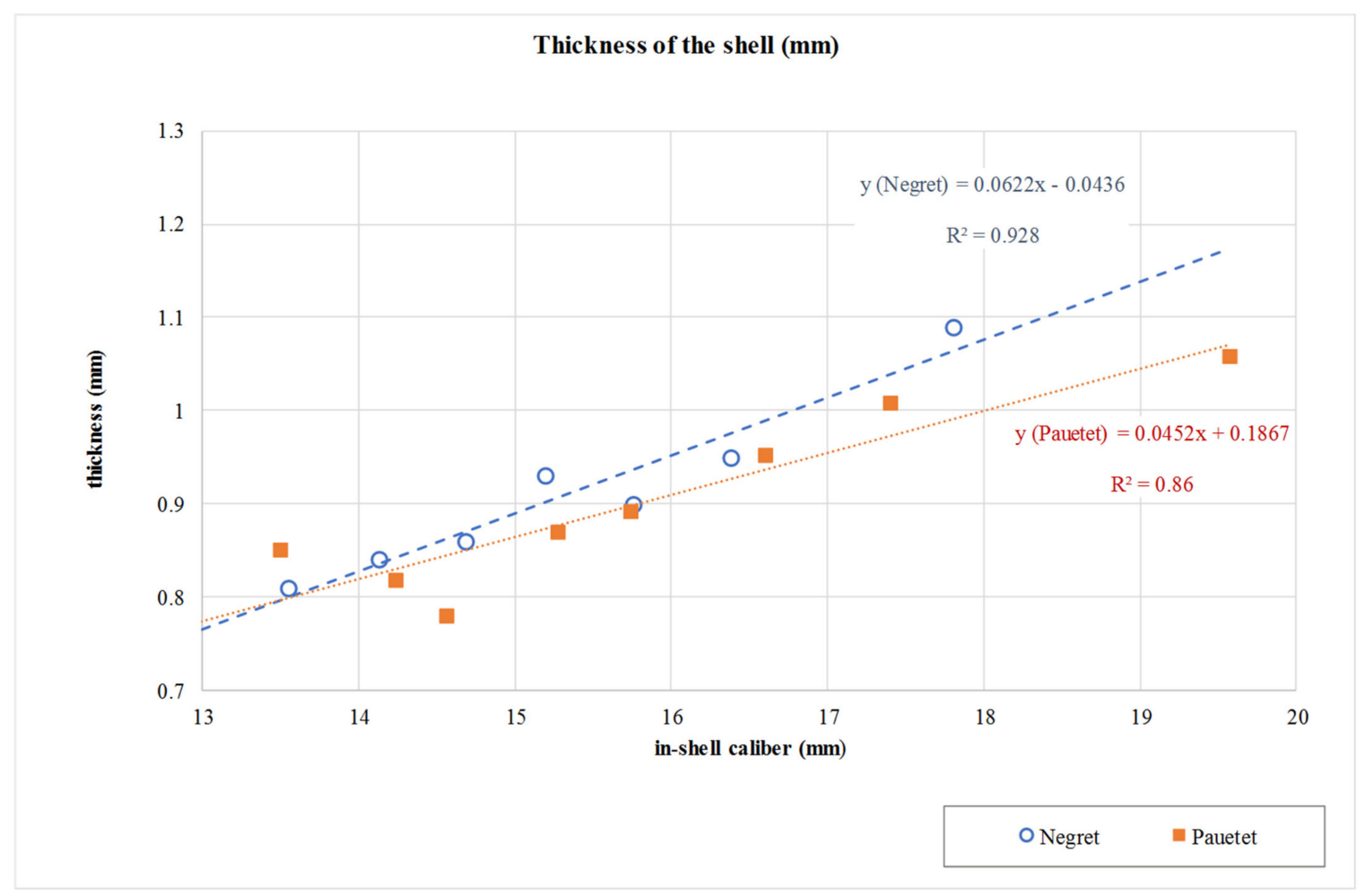

Figure 3. Linear models for central shell thickness of cv "Negret" and "Pauetet".

Significant differences between cultivars were observed for shell moisture, with a slightly higher water content in cv "Pauetet" (14.7\% in average) than for "Negret" (13.9\%), as shown in Table S2. However, shell moisture was not related to fruit size (Table 1).

Finally, kernel yield was higher for cv "Pauetet" ( $48.6 \%$ in average) than for cv "Negret" $(46.9 \%)$, as shown in Table S2. This trait decreased with nut size following a linear trend without significant differences between cultivars (Table 1 and Figure 4).

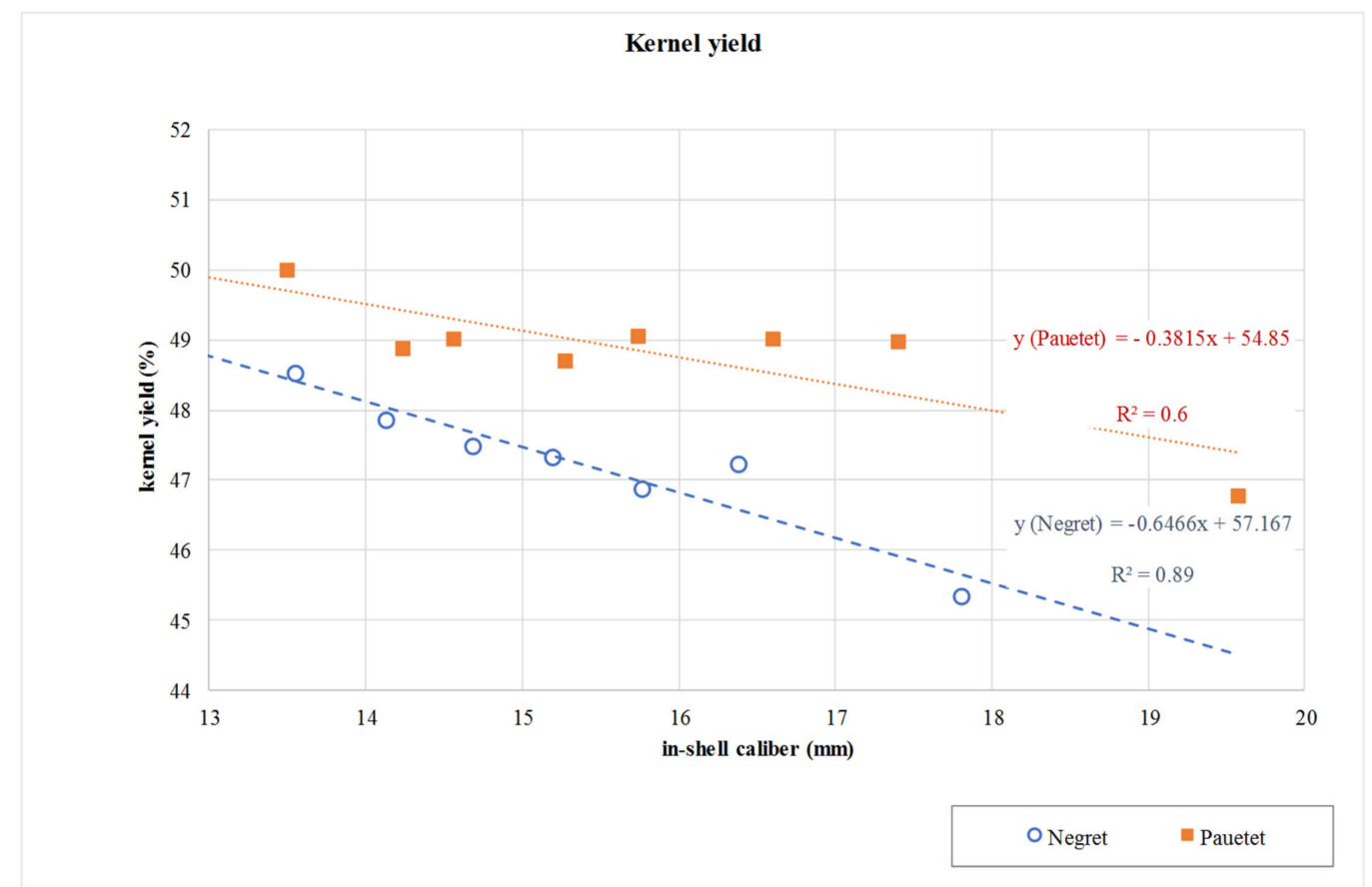

Figure 4. Linear models for kernel yield (\%) of cv "Negret" and "Pauetet". 


\subsection{Shelled Hazelnuts}

As occurred with in-shell nuts, the kernel roundness index decreased with caliber (Table S3), without any relation to hazelnut cultivar (Table 2 and Figure 5). The decreasing trend fits very well to a linear model $\left(\mathrm{R}^{2}>0.80 ; p<0.0001\right)$. Furthermore, kernel thickness increases faster than length with caliber. As a result, the roundness index tended to one for bigger kernels of the same cultivar.

Table 2. Hazelnut shelled nut traits of cv "Negret" and "Pauetet". Interpolated linear models and statistical tests for slope and cultivar homogeneity (Lightness: $\mathrm{L}^{*}$; reddish: $a^{*}$; and yellowish: $b^{*}$ ).

\begin{tabular}{|c|c|c|c|c|c|c|c|c|c|c|c|c|c|}
\hline \multirow{2}{*}{ Nut Trait } & \multirow{2}{*}{ Cultivar } & \multicolumn{8}{|c|}{ Linear Models } & \multicolumn{2}{|c|}{ Test for Slope } & \multicolumn{2}{|c|}{ Test for Cultivar } \\
\hline & & Intercep & & & Slope & & $\mathbf{R}^{2}$ & F Model & P Model & F Slope & P Slope & F Cultivar & P Cultivar \\
\hline \multirow{2}{*}{$\begin{array}{l}\text { Kernel length } \\
\quad(\mathrm{mm})\end{array}$} & Negret & 8.5987 & \pm & 0.6457 & 0.4231 & 0.0618 & 0.824 & 46.82 & $<0.0001$ & \multirow{2}{*}{0.98} & \multirow{2}{*}{0.3348} & \multirow{2}{*}{0.07} & \multirow{2}{*}{0.7917} \\
\hline & Pauetet & 7.3151 & \pm & 1.0799 & 0.5386 & 0.1013 & 0.739 & 28.28 & 0.0003 & & & & \\
\hline \multirow{2}{*}{$\begin{array}{l}\text { Kernel } \\
\text { thickness } \\
(\mathrm{mm})\end{array}$} & Negret & 1.9348 & \pm & 0.5645 & 0.7402 & 0.0540 & 0.949 & 187 & $<0.0001$ & \multirow{2}{*}{2.99} & \multirow{2}{*}{0.0991} & \multirow{2}{*}{2.31} & \multirow{2}{*}{0.1442} \\
\hline & Pauetet & 0.3774 & \pm & 0.3848 & 0.8546 & 0.0361 & 0.982 & 560 & $<0.0001$ & & & & \\
\hline \multirow{2}{*}{$\begin{array}{l}\text { Kernel } \\
\text { roundness }\end{array}$} & Negret & 2.0824 & \pm & 0.076 & $-0.0730 \pm$ & 0.0073 & 0.909 & 100 & $<0.0001$ & \multirow{2}{*}{0.07} & \multirow{2}{*}{0.8007} & \multirow{2}{*}{0.38} & \multirow{2}{*}{0.5427} \\
\hline & Pauetet & 2.0521 & \pm & 0.1151 & $-0.0697 \pm$ & 0.0107 & 0.807 & 41.75 & $<0.0001$ & & & & \\
\hline \multirow{2}{*}{$L^{*}$} & Negret & 26.07 & \pm & 2.67 & $0.1124 \quad \pm$ & 0.2554 & 0.019 & 0.19 & 0.6692 & \multirow{2}{*}{0.03} & \multirow{2}{*}{0.8652} & \multirow{2}{*}{5.08} & \multirow{2}{*}{0.0356} \\
\hline & Pauetet & 24.56 & \pm & 7.67 & $-0.0151 \pm$ & 0.7191 & 0.000 & 0.00 & 0.9836 & & & & \\
\hline \multirow{2}{*}{$a^{*}$} & Negret & 6.9783 & \pm & 0.7856 & 0.0997 & 0.0752 & 0.149 & 1.76 & 0.2147 & \multirow{2}{*}{0.08} & \multirow{2}{*}{0.7821} & \multirow{2}{*}{2.80} & \multirow{2}{*}{0.1098} \\
\hline & Pauetet & 6.9774 & \pm & 2.0004 & $0.0444 \quad \pm$ & 0.1876 & 0.006 & 0.06 & 0.8125 & & & & \\
\hline \multirow{2}{*}{$b^{*}$} & Negret & 13.86 & \pm & 1.45 & $-0.0841 \pm$ & 0.1396 & 0.035 & 0.36 & 0.5602 & \multirow{2}{*}{0.05} & \multirow{2}{*}{0.8228} & & \\
\hline & Pauetet & 13.69 & \pm & 4.69 & $-0.1859 \pm$ & 0.4404 & 0.017 & 0.18 & 0.6829 & & & 2.79 & 0.1104 \\
\hline Hus onclo $(0)$ & Negret & 1.1065 & \pm & 0.0441 & $-0.0086 \pm$ & 0.0042 & 0.291 & 4.11 & 0.0700 & 000 & (2) & 75 & ברת ת ח \\
\hline Hue angie ( ) & Pauetet & 1.0953 & \pm & 0.08 & $-0.0090 \pm$ & 0.0075 & 0.128 & 1.47 & 0.2536 & 0.00 & 0.9500 & 1.75 & 0.2023 \\
\hline & Negret & 15.48 & \pm & 1.51 & $-0.0208 \pm$ & 0.1447 & 0.002 & 0.02 & 0.8886 & & & & \\
\hline Chroma & Pauetet & 15.33 & \pm & 4.98 & $-0.1346 \pm$ & 0.4670 & 0.008 & 0.08 & 0.7790 & 0.06 & 0.8127 & 2.89 & 0.1048 \\
\hline
\end{tabular}

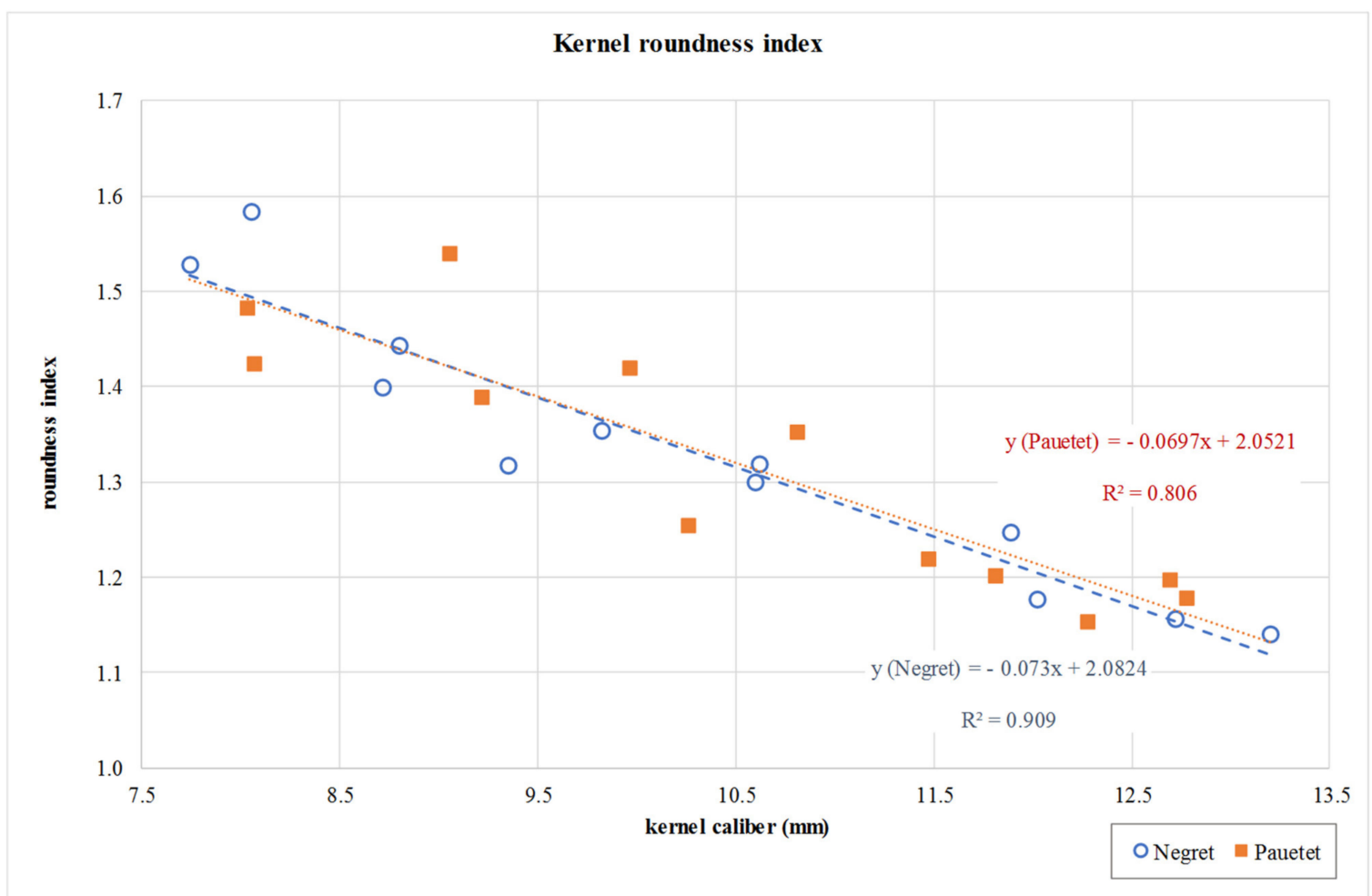

Figure 5. Linear models for kernel roundness index of cv "Negret" and "Pauetet". 
Regarding kernel skin color, no significant trend with respect to caliber was observed for either "Negret" or "Pauetet". Apart from a small difference in lightness $\left(L^{*}\right)$, which was higher in "Negret", the other color components $\left(a^{*}, b^{*}\right.$, Hue angle and Chroma) were equivalent for both cultivars (Table 2).

Roasting (R1) and blanching (B1) indexes (Table 3) were significantly different for each cultivar (R1 $=66.2 \%$ and B1 $=40.0 \%$ for "Negret", whereas $\mathrm{R} 1=56.1 \%$ and B1 $=21.0 \%$ for "Pauetet"). Additionally, "Negret" fit to a linear trend with caliber $\left(\mathrm{R}^{2}=0.58 ; p=0.0041\right.$ for $\mathrm{R} 1$ index and $\mathrm{R}^{2}=0.87 ; p<0.0001$ for B1 index), whereas "Pauetet" did not change with caliber. Figure 6 shows the different behavior of each cultivar for the B1 index.

Table 3. Hazelnut shelled nut industrial properties of cv "Negret" and "Pauetet". Interpolated linear models and statistical tests for slope and cultivar homogeneity. Roasting index R1 (\% of nuts peeled in more than 95\%); roasting index R2 (\% of nuts peeled in more than 50\%); blanching index B1 (\% of nuts peeled in more than 95\%); blanching index B2 (\% of nuts peeled in more than $50 \%$ ).

\begin{tabular}{|c|c|c|c|c|c|c|c|c|c|c|c|c|}
\hline \multirow{4}{*}{$\begin{array}{c}\text { Nut Trait } \\
\text { Roasting } \\
\text { aptitude R1 } \\
\begin{array}{c}(\%) \\
\text { (\%) }\end{array}\end{array}$} & \multirow{3}{*}{$\begin{array}{l}\text { Cultivar } \\
\text { Negret }\end{array}$} & \multicolumn{7}{|c|}{ Linear Models } & \multicolumn{2}{|c|}{ Test for Slope } & \multicolumn{2}{|c|}{ Test for Cultivar } \\
\hline & & \multicolumn{2}{|c|}{ Intercept } & \multicolumn{2}{|c|}{ Slope } & \multirow{2}{*}{$\begin{array}{l}\mathbf{R}^{\mathbf{2}} \\
0.578\end{array}$} & \multirow{2}{*}{$\begin{array}{c}\text { F Model } \\
13.71\end{array}$} & \multirow{2}{*}{$\begin{array}{c}\text { P Model } \\
0.0041\end{array}$} & \multirow{3}{*}{$\begin{array}{c}\text { F Slope } \\
5.25\end{array}$} & \multirow{3}{*}{$\begin{array}{c}\text { P Slope } \\
0.0342\end{array}$} & \multirow{3}{*}{$\begin{array}{c}\text { F Cultivar } \\
7.88\end{array}$} & \multirow{3}{*}{$\begin{array}{c}\text { P Cultivar } \\
0.0117\end{array}$} \\
\hline & & 0.0241 & \pm 0.1747 & 0.0619 & 0.0167 & & & & & & & \\
\hline & Pauetet & 0.4953 & \pm 0.1397 & 0.0059 & 0.0126 & 0.027 & 0.22 & 0.6486 & & & & \\
\hline \multirow{2}{*}{$\begin{array}{c}\text { Roasting } \\
\text { aptitude R2 } \\
(\%)\end{array}$} & Negret & 0.9317 & \pm 0.0582 & 0.0027 & 0.0056 & 0.022 & 0.23 & 0.6424 & \multirow{2}{*}{0.11} & \multirow{2}{*}{0.7470} & \multirow{2}{*}{22.69} & \multirow{2}{*}{0.0002} \\
\hline & Pauetet & 0.8233 & \pm 0.0934 & 0.0059 & 0.0084 & 0.059 & 0.50 & 0.4986 & & & & \\
\hline \multirow{2}{*}{$\begin{array}{c}\text { Blanching } \\
\text { aptitude B1 } \\
(\%)\end{array}$} & Negret & $-0.5632 \pm$ & \pm 0.1206 & 0.0936 & 0.0115 & 0.868 & 65.64 & $<0.0001$ & \multirow{2}{*}{22.07} & \multirow{2}{*}{0.0002} & \multirow{2}{*}{46.54} & \multirow{2}{*}{$<0.0001$} \\
\hline & Pauetet & 0.1612 & \pm 0.1538 & 0.0044 & 0.0138 & 0.013 & 0.10 & 0.7574 & & & & \\
\hline \multirow{2}{*}{$\begin{array}{c}\text { Blanching } \\
\text { aptitude B2 } \\
(\%)\end{array}$} & Negret & 0.1168 & \pm 0.2376 & 0.0648 & 0.0227 & 0.448 & 8.11 & 0.0173 & \multirow{2}{*}{0.03} & \multirow{2}{*}{0.8620} & \multirow{2}{*}{48.41} & \multirow{2}{*}{$<0.0001$} \\
\hline & Pauetet & $-0.2084 \pm$ & $\pm \quad 0.1944$ & 0.0589 & 0.0179 & 0.574 & 10.76 & 0.0112 & & & & \\
\hline \multirow{2}{*}{$\begin{array}{l}\text { Oil acidity (\% } \\
\text { ocelic acid) }\end{array}$} & Negret & 0.3219 & $\pm \quad 0.1109$ & $-0.0094 \pm$ & 0.0106 & 0.073 & 0.79 & 0.3961 & \multirow{2}{*}{1.56} & \multirow{2}{*}{0.2291} & \multirow{2}{*}{1.18} & \multirow{2}{*}{0.2918} \\
\hline & Pauetet & 0.7559 & \pm 0.3366 & $-0.0436 \pm$ & 0.0297 & 0.234 & 2.14 & 0.1865 & & & & \\
\hline \multirow{2}{*}{$\begin{array}{l}\text { Water activity } \\
\qquad\left(a_{\mathrm{w}}\right)\end{array}$} & Negret & 0.6695 & \pm 0.0297 & $-0.0033 \pm$ & 0.0028 & 0.117 & 1.33 & 0.2764 & \multirow{2}{*}{0.61} & 0 P & 107 & \\
\hline & Pauetet & 0.0441 & \pm 0.0561 & 0.0013 & 0.0053 & 0.006 & 0.06 & 0.8078 & & 0.4433 & 4.97 & $0.03 / 4$ \\
\hline
\end{tabular}

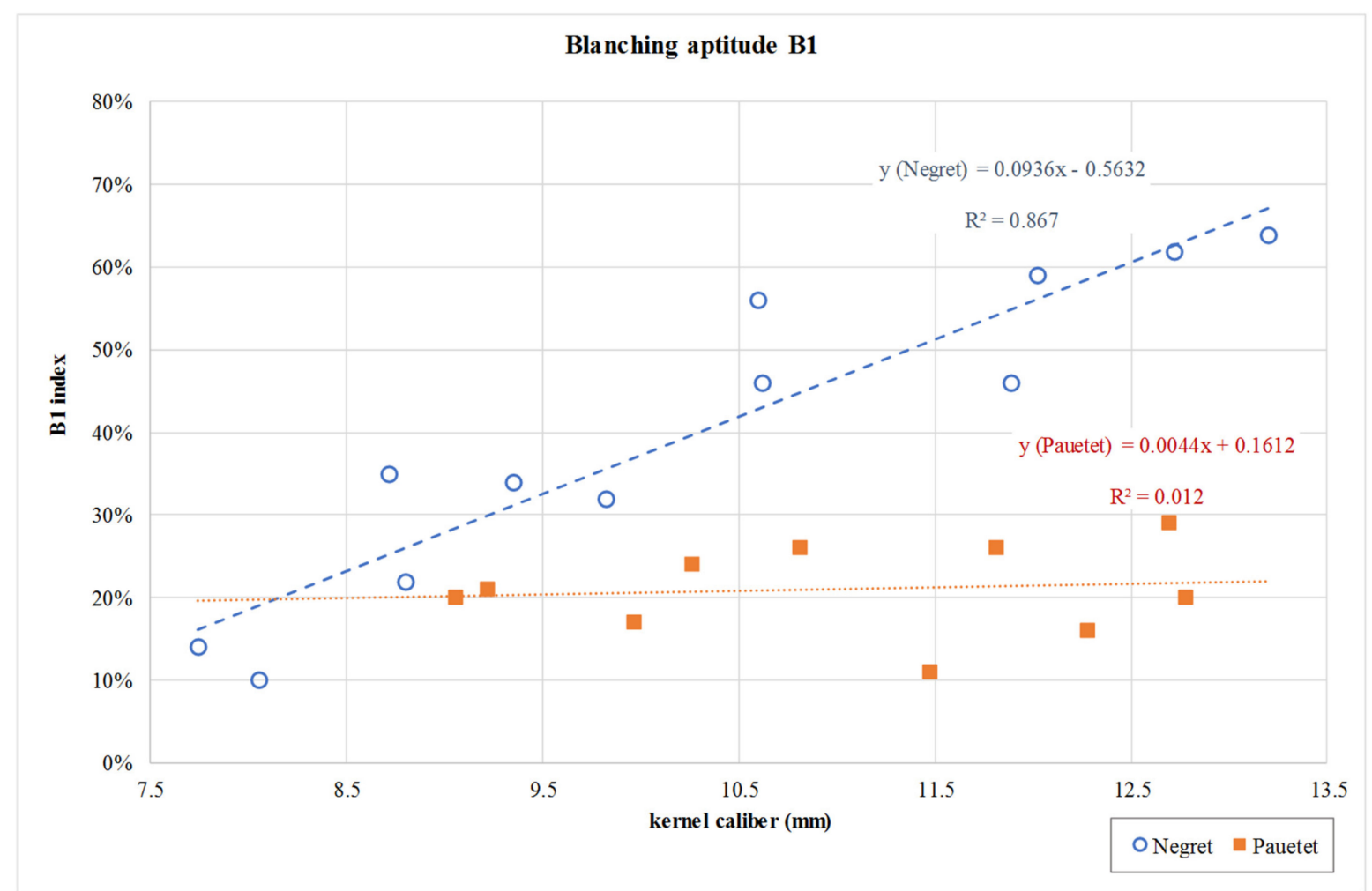

Figure 6. Linear models for kernel blanching (B1 index) of cv "Negret" and "Pauetet". 
Water activity (Tables 3 and S4) was slightly higher in cv "Pauetet" (0.658) than cv "Negret" (0.636), in accordance with the observed drift in moisture. Water activity did not show a linear trend with caliber, suggesting that differences in moisture were not due to external water absorption but to tissue water content. As stated above, kernel moisture was significantly higher for "Pauetet" (5.0\% in average) than for "Negret" $(4.7 \%)$. Furthermore, moisture decreased with caliber without significant differences between cultivars (Table 4 and Table S4). Though the linear model was significant, the adjustment was not very high, as shown in Figure $7\left(R^{2}=0.66\right.$ for "Negret" and $R^{2}=0.40$ for "Pauetet").

Table 4. Hazelnut shelled nut chemical composition of cv "Negret" and "Pauetet". Interpolated linear models and statistical tests for slope and cultivar homogeneity.

\begin{tabular}{|c|c|c|c|c|c|c|c|c|c|c|c|c|}
\hline \multirow{4}{*}{$\begin{array}{c}\text { Nut Trait } \\
\text { Moisture } \\
\text { content (\%) }\end{array}$} & \multirow{3}{*}{$\begin{array}{l}\text { Cultivar } \\
\text { Negret }\end{array}$} & \multicolumn{7}{|c|}{ Linear Models } & \multicolumn{2}{|c|}{ Test for Slope } & \multicolumn{2}{|c|}{ Test for Cultivar } \\
\hline & & \multicolumn{2}{|c|}{ Intercept } & \multicolumn{2}{|c|}{ Slope } & \multirow{2}{*}{$\begin{array}{l}\mathbf{R}^{2} \\
0.659\end{array}$} & \multirow{2}{*}{$\begin{array}{c}\text { F Model } \\
19.36\end{array}$} & \multirow{2}{*}{$\begin{array}{c}\text { P Model } \\
0.0013\end{array}$} & \multirow{3}{*}{$\begin{array}{c}\text { F Slope } \\
0.30\end{array}$} & \multirow{3}{*}{$\begin{array}{c}\text { P Slope } \\
0.5905\end{array}$} & \multirow{3}{*}{$\begin{array}{c}\text { F Cultivar } \\
5.72\end{array}$} & \multirow{3}{*}{$\begin{array}{c}\text { P Cultivar } \\
0.0267\end{array}$} \\
\hline & & 6.7179 & \pm 0.4626 & $-0.1949 \pm$ & 0.0443 & & & & & & & \\
\hline & Pauetet & 6.639 & \pm 0.6408 & $-0.1544 \pm$ & 0.0600 & 0.398 & 6.60 & 0.0279 & & & & \\
\hline \multirow{2}{*}{$\begin{array}{l}\text { Fat content } \\
\qquad(\%)\end{array}$} & Negret & 38.05 & $\pm \quad 6.72$ & $2.4105 \pm$ & 0.6308 & 0.619 & 14.60 & 0.0041 & \multirow{2}{*}{0.00} & \multirow{2}{*}{0.9673} & \multirow{2}{*}{1.70} & \multirow{2}{*}{0.2086} \\
\hline & Pauetet & 40.67 & \pm 6.06 & $2.3747 \quad \pm$ & 0.5795 & 0.651 & 16.79 & 0.0027 & & & & \\
\hline \multirow{2}{*}{ Protein $(\%)$} & Negret & 16.06 & $\pm \quad 0.97$ & $-0.1059 \pm$ & 0.0903 & 0.314 & 1.38 & 0.3254 & \multirow{2}{*}{3.28} & \multirow{2}{*}{0.1202} & \multirow{2}{*}{0.24} & \multirow{2}{*}{0.6439} \\
\hline & Pauetet & 20.29 & $\pm \quad 1.74$ & $-0.4268 \pm$ & 0.1588 & 0.707 & 7.22 & 0.0746 & & & & \\
\hline \multirow{2}{*}{$\begin{array}{c}\text { Crude fiber } \\
(\%)\end{array}$} & Negret & 14.6116 & \pm 2.6437 & $-0.2714 \pm$ & 0.2419 & 0.290 & 1.23 & 0.3488 & \multirow{2}{*}{4.99} & \multirow{2}{*}{0.0669} & \multirow{2}{*}{0.01} & \multirow{2}{*}{0.9360} \\
\hline & Pauetet & 5.0274 & \pm 3.4554 & $0.6109 \pm$ & 0.3130 & 0.559 & 3.81 & 0.1460 & & & & \\
\hline \multirow{2}{*}{ Sugars (\%) } & Negret & 5.2274 & \pm 0.7489 & $-0.2109 \pm$ & 0.0694 & 0.755 & 9.24 & 0.0559 & \multirow{2}{*}{0.51} & \multirow{2}{*}{0.5027} & \multirow{2}{*}{3.34} & \multirow{2}{*}{0.1173} \\
\hline & Pauetet & 5.6912 & \pm 1.2988 & $-0.3061 \pm$ & 0.1187 & 0.689 & 6.65 & 0.0818 & & & & \\
\hline \multirow{2}{*}{ Minerals (\%) } & Negret & 2.2106 & \pm 0.4259 & $-0.0135 \pm$ & 0.0395 & 0.038 & 0.12 & 0.7543 & \multirow{2}{*}{0.86} & & & \\
\hline & Pauetet & 1.4651 & \pm 0.6650 & $0.0522 \pm$ & 0.0607 & 0.197 & 0.74 & 0.4538 & & 0.3900 & 0.12 & $0.14 \angle 4$ \\
\hline
\end{tabular}

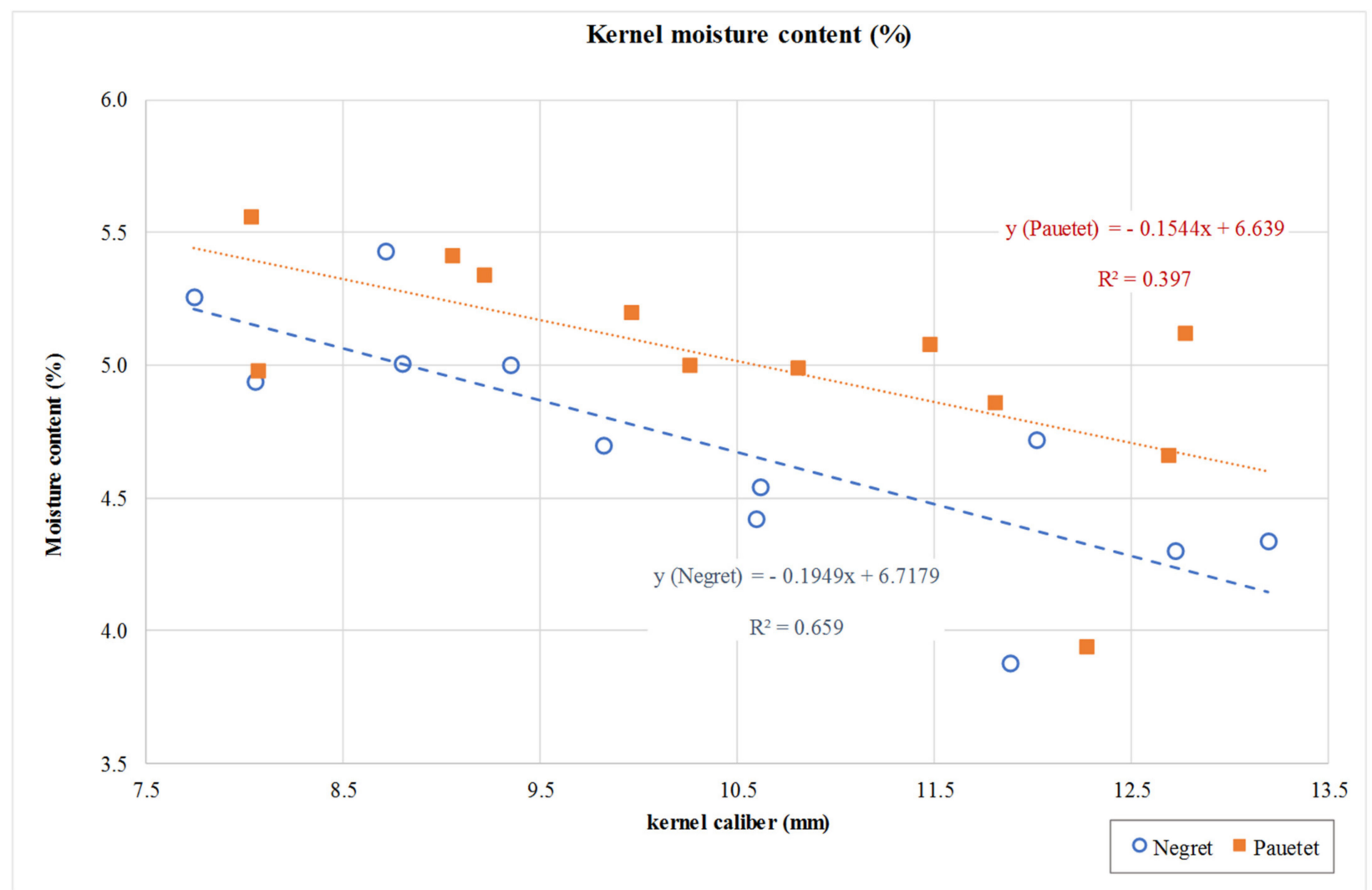

Figure 7. Linear models for kernel moisture content of cv "Negret" and "Pauetet". 
Regarding kernel chemical composition (Tables 4 and S4), no significant differences were observed between cultivars. However, the fat content fitted relatively well to a linear model related to caliber with a positive slope $\left(\mathrm{R}^{2}=0.63 ; p<0.0041\right.$ for "Negret" and $\mathrm{R}^{2}=0.65$; $p=0.0027$ for "Pauetet") without any differences in trend between cultivars (Figure 8).

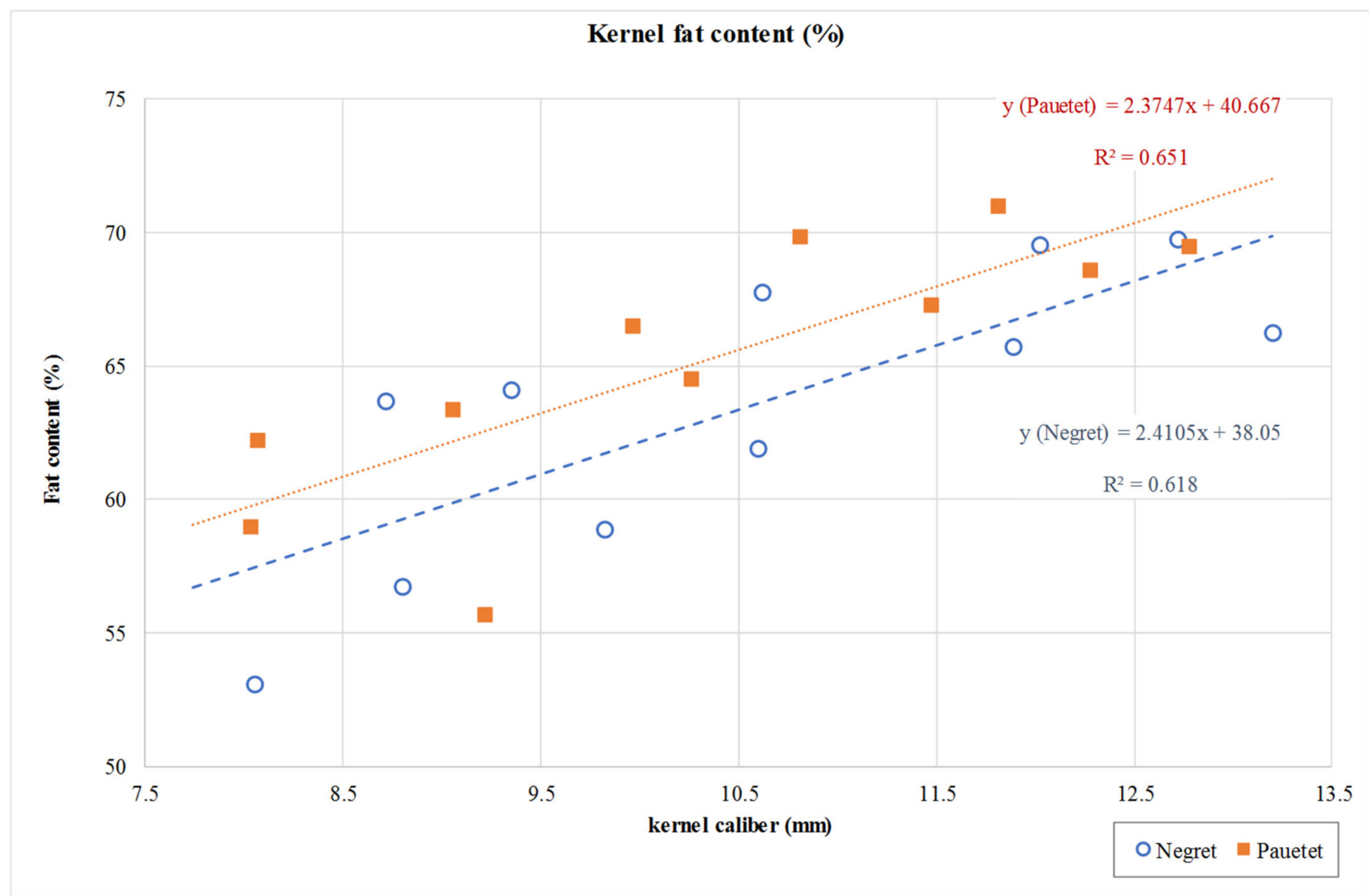

Figure 8. Linear models for kernel fat content of cv "Negret" and "Pauetet".

On the other hand, the total sugar content showed a significant linear trend with a negative slope $\left(R^{2}=0.76 ; p<0.0559\right.$ for "Negret" and $R^{2}=0.69 ; p<0.0818$ for "Pauetet" $)$ and no significant differences in trend between cultivars (Figure 9). $\alpha$-tocopherol fit to a linear model with a positive slope for "Negret" $\left(R^{2}=0.91 ; p<0.0112\right)$ but not for "Pauetet". However, the slope was positive in both cultivars (Figure 10). Finally, protein, crude fiber and mineral content did not show any significant relationship with kernel caliber.

The fatty acid composition, especially unsaturated and polyunsaturated fatty acids, fitted very well to linear models, with significant differences between cultivars concerning mean values but not for slopes (Table 5 and Table S4). In fact, the mean oleic acid content was higher in "Pauetet" (79.6\% in average) than "Negret" $(75.8 \%)$, and both cultivars showed a significant direct relationship with caliber, as shown in Figure $11\left(R^{2}>0.96\right.$; $p<0.0001)$. Linoleic acid content was significantly lower in "Pauetet" $(11.9 \%)$ than in "Negret" $(16.2 \%)$, and both cultivars followed a decreasing linear model with caliber $\left(R^{2}>0.97 ; p<0.0001\right)$. Then, linolenic acid was significantly higher in "Negret" $(0.09 \%)$ than "Pauetet" $(0.08 \%)$, and both cultivars followed a decreasing linear model with caliber $\left(R^{2}>0.85 ; p<0.0003\right)$. On the other hand, palmitic acid was significantly higher in "Pauetet" $(5.9 \%)$ than in "Negret" (5.7\%), but only "Pauetet" showed a significant increasing linear trend with caliber $\left(\mathrm{R}^{2}=0.64 ; p<0.0099\right)$, whereas "Negret" seemed to follow a similar trend, but the adjustment was poor $\left(R^{2}=0.07 ; p<0.4291\right)$. 


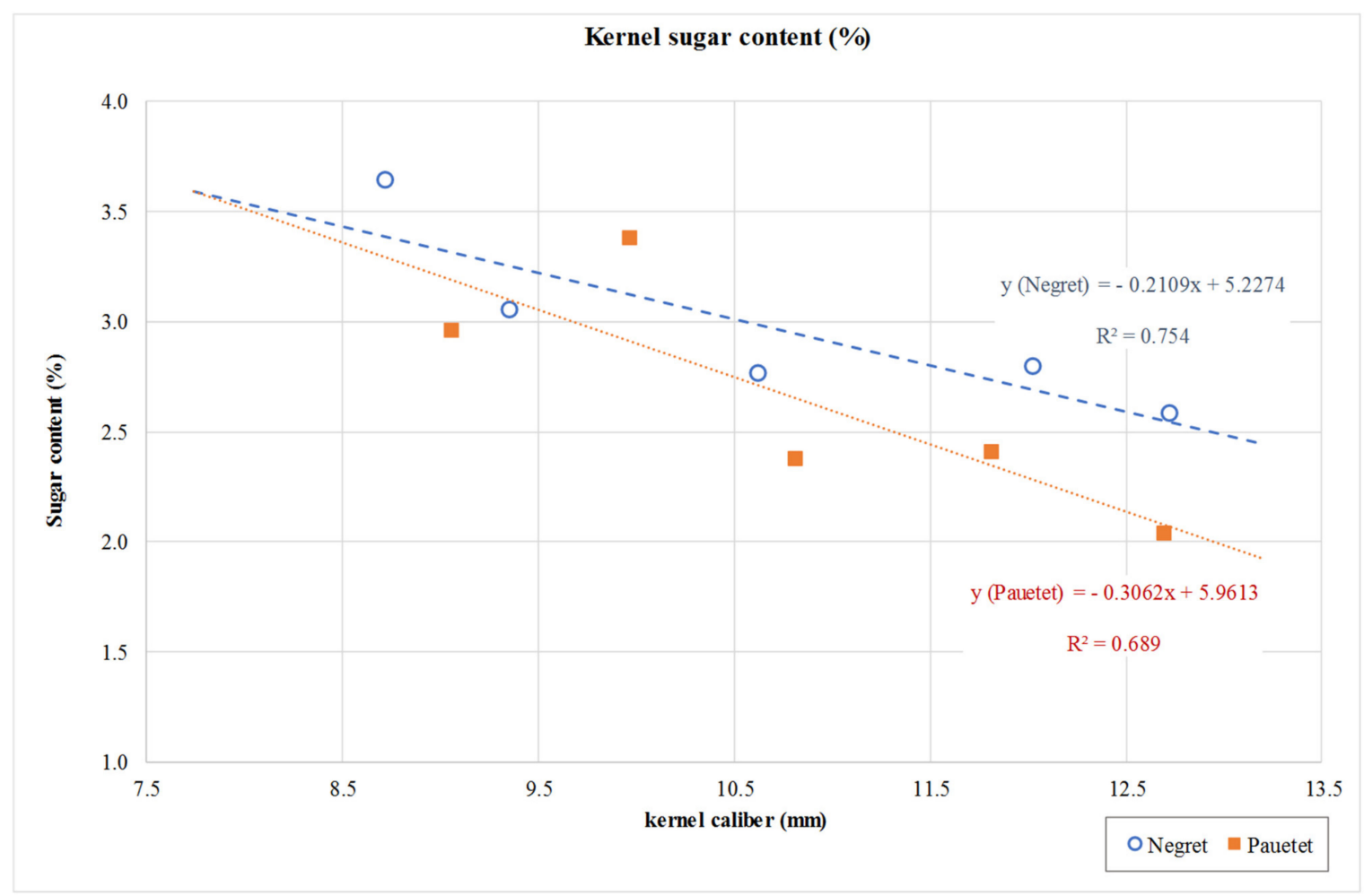

Figure 9. Linear models for kernel sugar content of cv "Negret" and "Pauetet".

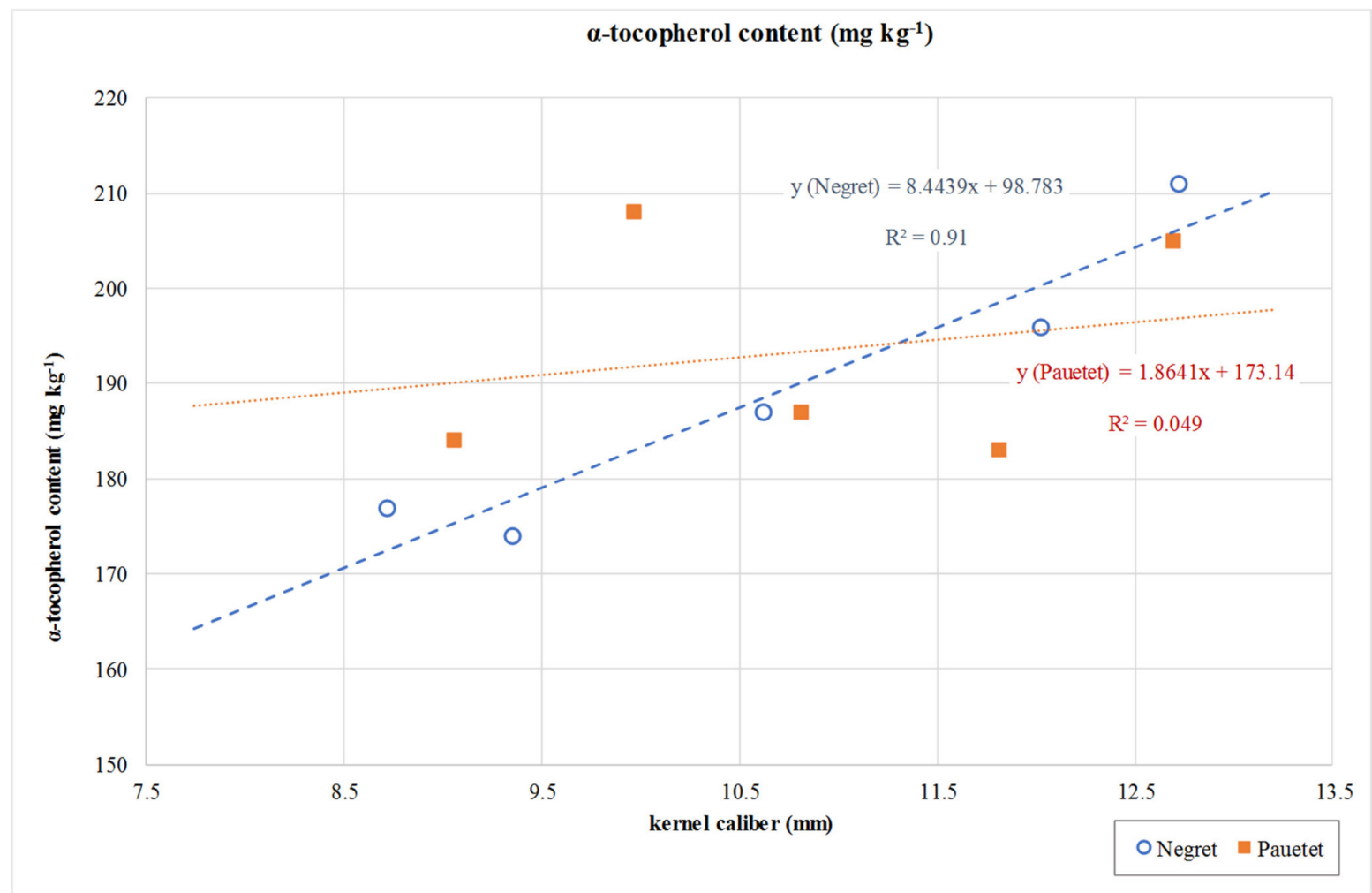

Figure 10. Linear models for $\alpha$-tocopherol content of cv "Negret" and "Pauetet". 
Table 5. Fatty acid composition, oil stability and $\alpha$-tocopherol content of cv "Negret" and "Pauetet". Interpolated linear models and statistical tests for slope and cultivar homogeneity.

\begin{tabular}{|c|c|c|c|c|c|c|c|c|c|c|c|c|}
\hline \multirow{4}{*}{$\begin{array}{c}\text { Nut Trait } \\
\begin{array}{c}\text { Palmitic acid } \\
(\mathrm{C} 16: 0 \%)\end{array}\end{array}$} & \multirow{3}{*}{$\begin{array}{l}\text { Cultivar } \\
\text { Negret }\end{array}$} & \multicolumn{7}{|c|}{ Linear Models } & \multicolumn{2}{|c|}{ Test for Slope } & \multicolumn{2}{|c|}{ Test for Cultivar } \\
\hline & & \multicolumn{2}{|c|}{ Intercept } & \multicolumn{2}{|c|}{ Slope } & \multirow{2}{*}{$\begin{array}{l}\mathbf{R}^{2} \\
0.071\end{array}$} & \multirow{2}{*}{$\begin{array}{c}\text { F Model } \\
0.69\end{array}$} & \multirow{2}{*}{$\begin{array}{c}\text { P Model } \\
0.4291\end{array}$} & \multirow{3}{*}{$\begin{array}{c}\text { F Slope } \\
4.16\end{array}$} & \multirow{3}{*}{$\begin{array}{c}\text { P Slope } \\
0.0582\end{array}$} & \multirow{3}{*}{$\begin{array}{c}\text { F Cultivar } \\
70.57\end{array}$} & \multirow{3}{*}{$\begin{array}{c}\text { P Cultivar } \\
<0.0001\end{array}$} \\
\hline & & 5.5462 & \pm 0.1404 & 0.0109 & 0.0132 & & & & & & & \\
\hline & Pauetet & 5.2791 & \pm 0.1826 & 0.0566 & 0.0161 & 0.638 & 12.32 & 0.0099 & & & & \\
\hline \multirow{2}{*}{$\begin{array}{l}\text { Oleic acid } \\
(\mathrm{C} 18: 1 \%)\end{array}$} & Negret & 52.51 & \pm 1.26 & 2.2169 & 0.1186 & 0.975 & 349 & $<0.0001$ & \multirow{2}{*}{0.12} & \multirow{2}{*}{0.7375} & \multirow{2}{*}{172} & \multirow{2}{*}{$<0.0001$} \\
\hline & Pauetet & 53.86 & $\pm \quad 1.99$ & 2.2906 & 0.1763 & 0.960 & 168 & $<0.0001$ & & & & \\
\hline \multirow{2}{*}{$\begin{array}{l}\text { Linoleic acid } \\
\quad(\mathrm{C} 18: 2 \%)\end{array}$} & Negret & 40.54 & \pm 1.31 & $-2.3228 \pm$ & 0.1129 & 0.975 & 356 & $<0.0001$ & \multirow{2}{*}{0.28} & \multirow{2}{*}{0.6048} & \multirow{2}{*}{205} & \multirow{2}{*}{$<0.0001$} \\
\hline & Pauetet & 39.33 & $\pm \quad 1.96$ & $-2.4385 \pm$ & 0.1729 & 0.966 & 198 & $<0.0001$ & & & & \\
\hline \multirow{2}{*}{$\begin{array}{l}\text { Linolenic acid } \\
\text { (C18:3\%) }\end{array}$} & Negret & 0.1787 & \pm 0.0112 & $-0.0085 \pm$ & 0.0011 & 0.878 & 64.67 & $<0.0001$ & \multirow{2}{*}{0.18} & \multirow{2}{*}{0.6789} & \multirow{2}{*}{14.10} & \multirow{2}{*}{0.0017} \\
\hline & Pauetet & 0.1679 & \pm 0.0134 & $-0.0077 \pm$ & 0.0012 & 0.858 & 42.30 & 0.0003 & & & & \\
\hline \multirow{2}{*}{$\begin{array}{l}\text { Stability (h at } \\
\left.120{ }^{\circ} \mathrm{C}\right)\end{array}$} & Negret & $-0.0214 \pm$ & \pm 0.8942 & 0.6109 & 0.0841 & 0.854 & 52.80 & $<0.0001$ & \multirow{2}{*}{1.02} & \multirow{2}{*}{0.3269} & \multirow{2}{*}{28.01} & \multirow{2}{*}{$<0.0001$} \\
\hline & Pauetet & $-1.1723 \pm$ & \pm 1.6516 & 0.7770 & 0.1459 & 0.802 & 28.35 & 0.0011 & & & & \\
\hline \multirow{2}{*}{$\begin{array}{l}\alpha \text {-tocopherol } \\
(\mathrm{mg} / \mathrm{kg})\end{array}$} & Negret & 98.78 & \pm 16.24 & 8.4429 & 1.5048 & 0.913 & 31.49 & 0.0112 & \multirow{2}{*}{1.98} & 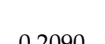 & 046 & 05241 \\
\hline & Pauetet & 173.14 & \pm 51.69 & 1.8664 & 4.7236 & 0.049 & 0.16 & 0.7195 & & 0.2090 & 0.46 & $0.5<41$ \\
\hline
\end{tabular}

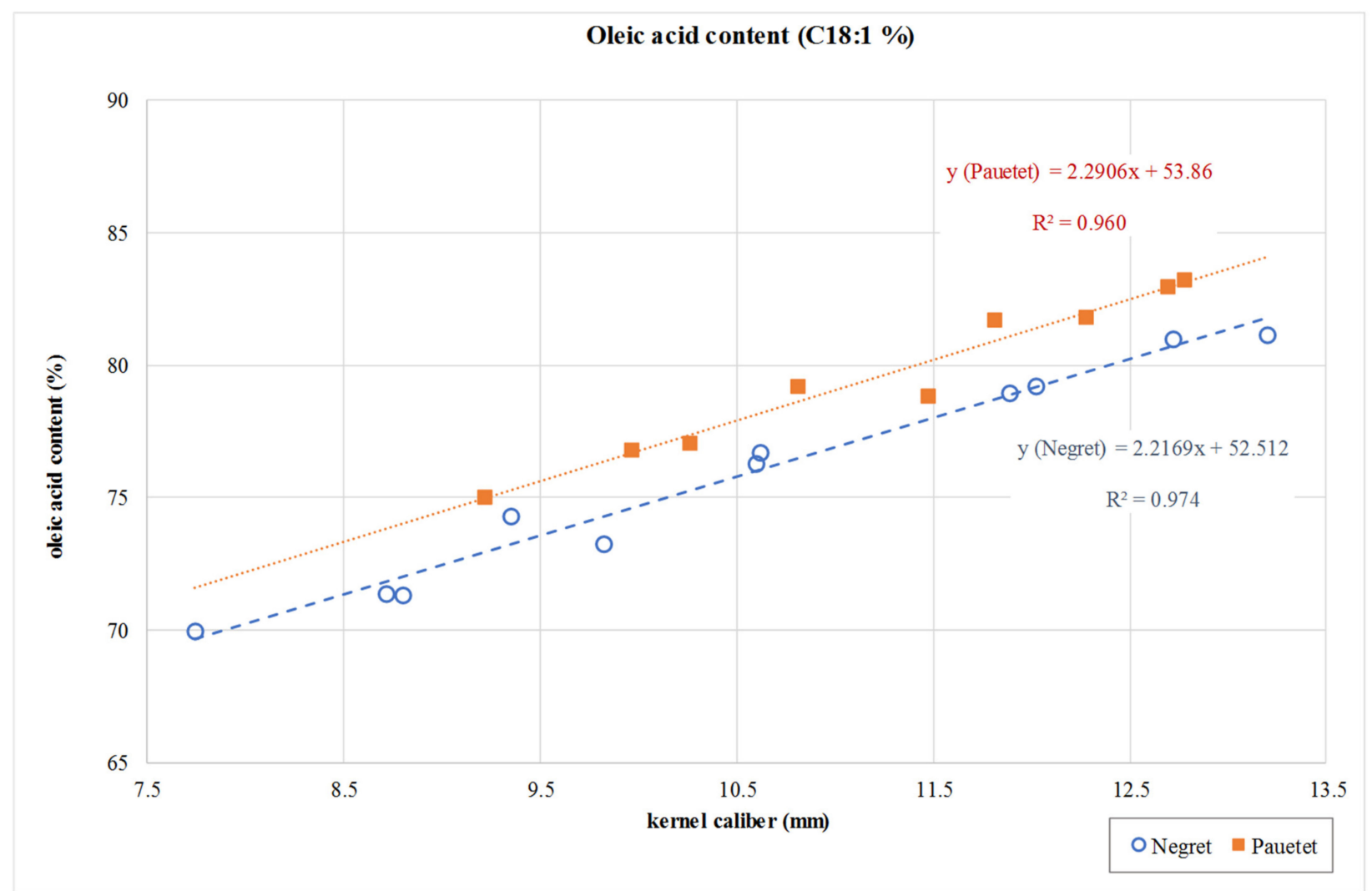

Figure 11. Linear models for oleic acid content of cv "Negret" and "Pauetet".

In agreement with the observed trends in polyunsaturated fatty acids, the mean oil stability was higher in "Pauetet" $\left(7.57 \mathrm{~h}\right.$ at $\left.120^{\circ} \mathrm{C}\right)$ than in "Negret" $\left(6.39 \mathrm{~h}\right.$ at $\left.120^{\circ} \mathrm{C}\right)$, and it fit to a linear increasing trend with caliber (Table 5 and Figure 12), which is equivalent for both cultivars $\left(\mathrm{R}^{2}>0.80 ; p<0.0011\right)$. 


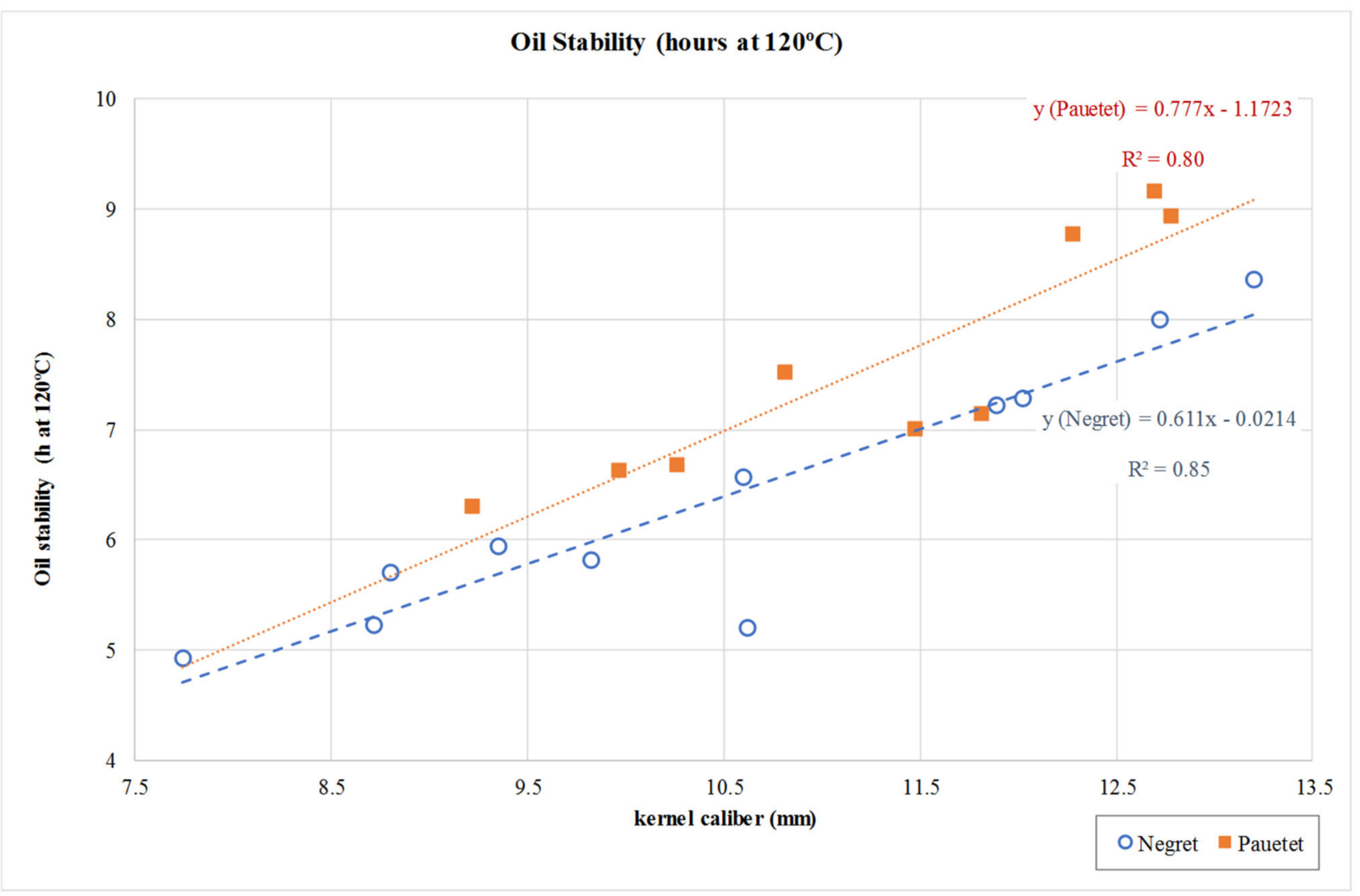

Figure 12. Linear models for oil stability of cv "Negret" and "Pauetet".

\section{Discussion and Conclusions}

The fact that the in-shell roundness index is closer to one for bigger nuts and that bigger fruits have a thicker shell, regardless of the cultivar, could be useful information for shelling machine builders. Cristofori et al. [16] also reported significant differences for nut roundness concerning years, but did not report any relationship with kernel caliber. In addition, the reported result that bigger kernels are more spherical is interesting for the chocolate industry, mainly for specialties in which a whole kernel is placed in the center, such as in the case of bonbons.

Concerning the roasting and blanching aptitude of both hazelnut cultivars, the results of this study agree with those reported by Rovira et al. [26] that "Negret" has a significantly better roasting aptitude than "Pauetet". Interestingly, in the present study, results show that if one cultivar (i.e., "Negret") is suitable for such processes, this property is enhanced with kernel caliber, whereas if the cultivar is unsuitable (i.e., "Pauetet"), this property negatively affects all the calibers.

Romero et al. [2] reported that "Negret" and "Pauetet" have similar chemical compositions, at least for major components, which agrees with the presented results for fat content, protein, fiber, sugar, minerals and tocopherol. However, significant differences were found for fatty acid composition. Rovira et al. $[25,26]$ reported the industrial traits and chemical composition of several hazelnut cultivars planted in Tarragona (Spain), including "Negret" and "Pauetet". These researchers observed significant differences among cultivars and years, mainly for fat and total sugar contents, but not for "Negret" and "Pauetet", which showed a very similar composition, in accordance with the results obtained in the present study. Similar results were reported in Italy by Cristofori et al. [16] for a different group of cultivars. Bacchetta et al. [41], in a study of 75 accessions of European hazelnut germplasm, also reported similar values of fat content and fatty acid composition for both cultivars, "Negret" and "Pauetet", over two years of study. On the other hand, Percerisa et al. [29] 
observed significant differences in chemical composition due to environmental factors for both cultivars over a three-year period. In the same vein, Klockmann et al. [42] developed classification models to decipher geographical origin using metabolomics approaches; however, they did not compare the same cultivar growing in different countries but only mixed samples from different countries.

To the best of our knowledge, the finding that chemical composition changes almost linearly with kernel caliber has not been previously reported. Xenia has positive effects on the quality of fruit concerning both nut size and chemical composition via crosspollination [31]. However, this does not explain the linearity with kernel size since xenia seems to have a qualitative effect. Furthermore, the results were the same for two years and two cultivars. This is more difficult to explain through the cross-pollination effect, which can change every year, especially in the Tarragona area where several cultivars are used as pollinizers. Finally, it must be stated that hazelnut is a self-incompatible species [43]; thus, cross-pollination happens in most fruit crops every year, and differences due to xenia are expected to be low.

Fat content variation in relation to kernel size can be very useful for the confectionery industry, which is concerned with this property. In fact, some technological food properties, such as final taste and consumer acceptability, improve with fat content [44,45]. For this purpose, choosing higher calibers for a given cultivar could be an interesting direction when there is a demand for better quality at a higher price. In some cases, fat migration is a problem [46,47]; thus, selecting kernels with lower fat content could be an option. On the other hand, the total energy of a product is sometimes a handicap, mainly when it includes sugar and fat from the hazelnut; in these cases, choosing kernels with a smaller caliber could help, since this reduces the fat content. The fact that the total sugar content of hazelnut increases at lower calibers should not be a big problem, since fat energy is more than two-fold higher than that of sugar, and our results show that the slope is ten-fold lower for the relationship of sugar with caliber than for fat content.

The positive increasing linear trend in oleic acid and stability with kernel size is a very interesting point, which has not been reported to date. In fact, differences among varieties were previously reported [16,17]. In addition, these researchers highlighted the influence of environmental conditions from either the yearly climate or geographical origin. However, no reports could be found relating fatty acid composition to kernel size. This is a very useful tool, because it enables the improvement of the final stability or monounsaturated fatty acid content through calibration.

Finally, these results demonstrate that hazelnut grading, considering both cultivars, 'Negret and "Pauetet", and nut size, can help to improve the total commercial value of the harvest, because the industry can benefit from different industrial properties.

Supplementary Materials: The following are available online at https:/ /www.mdpi.com/article/ 10.3390/agriculture11111115/s1, Table S1: In-shell fruit physical traits according to nut caliber; Table S2: In-shell fruit physical traits according to hazelnut cultivar; Table S3: Kernel characteristics according to caliber; Table S4: Kernel characteristics according to hazelnut cultivar.

Author Contributions: A.R.-A. designed and carried out the experiments and statistical analysis. M.R., V.C. and C.S. revised the results and helped with the discussion and bibliographical references. All authors have read and agreed to the published version of the manuscript.

Funding: The research was funded by Spanish-CDTI, PROJECT n.940263.

Data Availability Statement: The data presented in this study are available on request from the corresponding author.

Acknowledgments: Authors thanks COSELVA SCCL for providing samples and funding. A. Romero and M. Rovira acknowledge financial support from the CERCA Program from Generalitat of Catalonia.

Conflicts of Interest: The authors declare no conflict of interest. 


\section{References}

1. Batlle, I.; Aletà, N.; Rovira, M.; Torguet, L.; Miarnau, X.; Romero, M. Retos de futuro de los frutos secos en España. Vida Rural. 2018, 442, 70-78.

2. Romero, A.; Tous, J.; Plana, J.; Díaz, I.; Boatella, J.; García, J.; López, A. Commercial quality characterization of spanish "Negret” cultivar. Acta Hort. 1997, 445, 157-163. [CrossRef]

3. Islam, A.; Özkutlu, F.; Tonkaz, T.; Cristofori, V.; Silvestri, C.; Pica, A.L.; Speranza, S.; Rovira, M.; Romero, A.; Batlle, I. Hazelnut Cultivation in Europe; Islam, V.C.A., Mercé, R., Eds.; MKB Halk Kütüphanesi Yayinevi: Istambul, Turkey, 2018.

4. Radicati, L.; Romisondo, P.; Me, G. Le caratteristiche qualitative della nocciola in rapporto alle esigenze dell'industria dolciaria. In Atti del Convegno Nazionale Su il Miglioramento Della Coltura del Mandorlo e del Nocciolo. Aspetti Genetici e Tecnici; Regione Sicilia: Siracusa Messina, Messina, Italy, 1979; pp. 137-143.

5. Garrone, W.; Vacchetti, M. La qualità delle nocciole in rapporto alle esigenze dell'industria dolciaria utilizzatrice. Acta Hortic. 1994, 351, 641-648. [CrossRef]

6. Liepe, M. Taste variations for chocolate bars-filling mass, fruits, nuts. Zucker Suesswarenwirtschaft 1992, 45, $264-267$.

7. Voigt, J.; Wrann, D.; Heinrichs, H.; Biehl, B. The proteolytic formation of essential cocoa-specific aroma precursors depends on particular chemical structures of the vicilin-class globulin of the cocoa seeds lacking in the globular storage proteins of coconuts, hazelnuts and sunflower seeds. Food Chem. 1994, 51, 197-205. [CrossRef]

8. Ebrahem, K.S.; Richardson, D.G.; Tetley, R.M. Effects of storage temperature, kernel intactness and roasting temperature on vitamin E, fatty acids and peroxide value of hazelnuts. Acta Hortic. 1994, 351, 677-684. [CrossRef]

9. Perren, R.; Escher, F. Rösttechnologie von Haselnüssen, Teil 1: Einfluss von Produkttemperatur und Röstgrad auf die Oxidationsstabilität der gerösteten Nüsse. ZSV 1996, 1, 4.

10. Richardson, D.G.; Ebrahem, K. Hazelnut quality as affected by roasting temperatures and duration. Acta Hortic. 1997, 445, 301-304. [CrossRef]

11. Romero, A.; Tous, J.; Guerrero, L.; Gou, P.; Guardia, M.D. Aplicaciones del análisis sensorial en el tostado industrial de avellana en grano. Frutic. Prof. 1999, 104, 71-77.

12. Demir, A.D.; Frías, J.M.; Cronin, K.; Abodayeh, K. Modelling of the kinetics of colour change in hazelnuts during air roasting. J. Food Eng. 2002, 53, 283-292. [CrossRef]

13. Saklar, S.; Ungan, S.; Katnas, S. Microstructural changes in hazelnuts during roasting. Food Res. Int. 2003, 36, 19-23. [CrossRef]

14. Ruggeri, S.; Cappelloni, M.; Gambelli, L.; Nicoli, S.; Carnovale, E. Chemical composition and nutritive value of nuts grown in Italy. Ital. J. Food Sci. 1998, 10, 243-252.

15. Valentini, N.; Zeppa, G.; Rolle, L.; Me, G. Caratterizzazione chimico-fisica e sensoriale della nocciola Tonda Gentille delle Langhe. In Atti $2^{\circ}$ Convegno Naz. Sul Nocciolo; Regione Campania, Ed.; Ottobre: Giffoni Valle Piana, Italy, 2002; pp. $279-287$.

16. Cristofori, V.; Ferramondo, S.; Bertazza, G.; Bignami, C. Nut and kernel traits and chemical composition of hazelnut (Corylus avellana L.) cultivars. J. Sci. Food Agri. 2008, 88, 1091-1098. [CrossRef]

17. Parcerisa, J.; Rafecas, M.; Castellote, A.I.; Codony, R.; Farràn, A.; Garcia, J.; Lopez, A.; Romero, A.; Boatella, J. Influence of variety and geographical origin on the lipid fraction of hazelnuts (Coryllus avellana L.) from spain: (II). triglyceride composition. Food Chem. 2014, 50, 245-249. [CrossRef]

18. Ebrahem, K.S.; Richardson, D.G.; Tetley, R.M.; Mehlenbacher, S.A. Oil content, fatty acid composition and vitamin E concentration of 17 hazelnut varieties, compared to other types of nuts and oil seeds. Acta Hortic. 1994, 351, 685-692. [CrossRef]

19. Ayfer, M.; Uzun, A.; Bas, F. Turkish Hazelnut Cultivars; Black Sea Region Hazelnut Exporters Union: Ankara, Turkey, $1986 ;$ p. 95.

20. Król, K.; Gantner, M.; Piotrowska, A. Morphological traits, kernel composition and sensory evaluation of hazelnut (Corylus avellana L.) cultivars grown in Poland. Agronomy 2019, 9, 703. [CrossRef]

21. Król, K.; Gantner, M.; Piotrowska, A.; Hallmann, E. Effect of climate and roasting on polyphenols and tocopherols in the kernels and skin of six hazelnut cultivars (Corylus avellana L.). Agriculture 2021, 10, 36. [CrossRef]

22. Ciemniewska-Żytkiewicz, H.; Pasini, F.; Verardo, V.; Bryś, J.; Koczoń, P.; Caboni, M.F. Changes of the lipid fraction during fruit development in hazelnuts (Corylus avellana L.) grown in Poland. Eur. J. Lipid Sci. Technol. 2015, 117, 710-717. [CrossRef]

23. Ciemniewska-Zytkiewicz, H.; Verardo, V.; Pasini, F.; Bry's, J.; Koczo'n, P.; Caboni, M.F. Determination of lipid and phenolic fraction in two hazelnut (Corylus avellana L.) cultivars grown in Poland. Food Chem. 2015, 168, 615-622. [CrossRef] [PubMed]

24. Botta, R.; Gianotti, C.; Richardson, D.; Suwanagul, A.; Sanz, C.L. Hazelnut variety organic acids, sugars, and total lipid fatty acids. Acta Hortic. 1994, 351, 693-699. [CrossRef]

25. Rovira, M.; Romero, A.; Botta, R.; Di Giammatteo, V.; Duval, H.; Drogoudi, P.; Spera, A.; Silva, D.; Solar, A.; Bacchetta, L. Biochemical analysis in almond and hazelnut European cultivars. Ann. Nutr. Metab. 2013, 62, 82.

26. Rovira, M.; Hermoso, J.F.; Romero, A. Performance of hazelnut cultivars from Oregon, Italy, and Spain, in Northeastern Spain. HortTechnology 2017, 27, 631-638. [CrossRef]

27. Sarraquigne, J.P. Hazelnut production in France. Acta Hort. 2004, 686, 669-672. [CrossRef]

28. Parcerisa, J.; Boatella, J.; Codony, R.; Rafecas, M.; Castellote, A.I.; García, J.; López, A.; Romero, A. Comparison of fatty acid and triacylglycerol composition of different hazelnut varieties (Corylus avellana L.) cultivated in Catalonia (Spain). J. Agric. Food Chemistry 1995, 43, 13-16. [CrossRef] 
29. Parcerisa, J.; Rafecas, M.; Castellote, A.I.; Codony, R.; Farràn, A.; García, J.; Gonzalez, C.; López, A.; Romero, A.; Boatella, J. Influence of variety and geographical origin on the lipid fraction of hazelnuts (Corylus avellana L.) from Spain: (III) Oil stability, tocopherol content and some mineral contents (Mn, Fe, Cu). Food Chem. 1995, 53, 71-74. [CrossRef]

30. Silvestri, C.; Bacchetta, L.; Bellincontro, A.; Cristofori, V. Advances in cultivar choice, hazelnut orchard management, and nut storage to enhance product quality and safety: An overview. J. Sci. Food Agric. 2021, 101, 27-43. [CrossRef] [PubMed]

31. Fattahi, R.; Mohammadzedeh, M.; Khadivi-Khub, A. Influence of different pollen sources on nut and kernel characteristics of hazelnut. Sci. Hortic. 2014, 173, 15-19. [CrossRef]

32. Kodad, O.; Estopañán, G.; Juan, T.; Rafel Socias i Company. Xenia effects on oil content and fatty acid and tocopherol concentrations in autogamous almond cultivars. J. Agric. Food Chem. 2009, 57, 10809-10813. [CrossRef]

33. Rasouli, M.; Imani, A. Effect of supplementary pollination by different pollinizers on fruit set and nut physicochemical traits of 'Supernova', a self-compatible almond. Fruits 2016, 71, 299-306. [CrossRef]

34. Alizadeh-Salte, S.; Farhadi, N.; Arzani, K.; Khoshghalb, H. Almond oil quality as related to the type of pollen source in Iranian self-incompatible cultivars. Int. J. Fruit Sci. 2018, 18, 29-36. [CrossRef]

35. Kämper, W.; Thorp, G.; Wirthensohn, M.; Brooks, P.; Trueman, S.J. Pollen Paternity Can Affect Kernel Size and Nutritional Composition of Self-Incompatible and New Self-Compatible Almond Cultivars. Agronomy 2021, 11, 326. [CrossRef]

36. Zhang, X.-H.; Yuan, D.-Y.; Zou, F.; Fan, X.-M.; Tang, J.; Zhu, Z.-J. Studies on the pollen xenia of Castanea henryi. Acta Hortic. 2016, 43, 61-70. [CrossRef]

37. Acar, I.; Eti, S. Nut quality of 'Kirmizi', 'Siirt' and 'Ohadi' pistachio cultivars as affected by different pollinators. Acta Hortic. 2011, 912, 81-86. [CrossRef]

38. Richards, T.E.; Kämper, W.; Trueman, S.J.; Wallace, H.M.; Ogbourne, S.M.; Brooks, P.R.; Nichols, J.; Hosseini Bai, S. Relationships between nut size, kernel quality, nutritional composition and levels of outcrossing in three macadamia cultivars. Plants 2020, 9 , 228. [CrossRef] [PubMed]

39. Etheridge, R.D.; Pesti, G.M.; Foster, E.H. A comparison of nitrogen values obtained utilizing the Kjeldahl nitrogen and Dumas combustion methodologies (Leco CNS 2000) on samples typical of an animal nutrition analytical laboratory. Anim. Feed. Sci. Technol. 1998, 73, 21-28. [CrossRef]

40. AOCS Official Method Ce 8-89. Determination of tocopherols and tocotrienols in vegetable oils and fats by HPLC. In Official Methods and Recommended Practices of the American Oil Chemists' Society; AOCS Press: Champaign, IL, USA, $1997 ;$ p. 20.

41. Bacchetta, L.; Aramiri, M.; Zini, A.; Di Giammatteo, V.; Spera, D.; Drogiudi, P.; Rovira, M.; Silva, A.P.; Solar, A.; Botta, R. Fatty acids and alpha-tocopherol composition in hazelnut (Corylus avellana L.): A chemotrofic approach to emphasize the quality of European germplasm. Euphytica 2013, 191, 57-73. [CrossRef]

42. Klockmann, S.; Reiner, E.; Bachmann, R.; Hackl, T.; Fischer, M. Food Fingerprinting: Metabolomic Approaches for Geographical Origin Discrimination of Hazelnuts (Corylus avellana) by UPLC-QTOFMS. J. Agric. Food Chem. 2016, 64, 9253-9262. [CrossRef] [PubMed]

43. Thompson, M.M.; Lagerstedt, H.B.; Mehlenbacher, S.A. Hazelnuts. In Fruit Breeding, Volume III: Nuts; Jules, J., James, N., Eds.; Moore: New York, NY, USA, 1996; pp. 125-184.

44. Romero, A.; Tous, J.; Plana, J.; Guardia, M.D.; Díaz, I. How cultivar choice affects Spanish consumers' acceptance of marzipan and chocolates made with almonds. Acta Hortic. 2001, 591, 117-123. [CrossRef]

45. Romero, A.; Tous, J.; Plana, J. How cultivar choice affects Spanish consumers' acceptance of chocolates, bonbons and hard turron made with hazelnuts. Acta Hortic. 2005, 686, 577-584. [CrossRef]

46. Couzens, P.J.; Wille, H.J. Fat migration in composite confectionery products. Manuf. Confect. 1997, 77, 45-47.

47. Groves, R. Fat migration through chocolate. Candy Ind. 1999, 164, 28. [CrossRef] 